\title{
A systematic review of the impact of routine collection of patient reported outcome measures on patients, providers and health organisations in an oncologic setting
}

Jack Chen ${ }^{1,2^{*}}$, Lixin $\mathrm{Ou}^{1,2}$ and Stephanie J Hollis ${ }^{1,2}$

\begin{abstract}
Background: Despite growing interest and urges by leading experts for the routine collection of patient reported outcome (PRO) measures in all general care patients, and in particular cancer patients, there has not been an updated comprehensive review of the evidence regarding the impact of adopting such a strategy on patients, service providers and organisations in an oncologic setting.

Methods: Based on a critical analysis of the three most recent systematic reviews, the current systematic review developed a six-method strategy in searching and reviewing the most relevant quantitative studies between January 2000 and October 2011 using a set of pre-determined inclusion criteria and theory-based outcome indicators. The Grading of Recommendations, Assessment, Development, and Evaluation (GRADE) system was used to rate the quality and importance of the identified publications, and the synthesis of the evidence was conducted.

Results: The 27 identified studies showed strong evidence that the well-implemented PROs improved patientprovider communication and patient satisfaction. There was also growing evidence that it improved the monitoring of treatment response and the detection of unrecognised problems. However, there was a weak or non-existent evidence-base regarding the impact on changes to patient management and improved health outcomes, changes to patient health behaviour, the effectiveness of quality improvement of organisations, and on transparency, accountability, public reporting activities, and performance of the health care system.
\end{abstract}

Conclusions: Despite the existence of significant gaps in the evidence-base, there is growing evidence in support of routine PRO collection in enabling better and patient-centred care in cancer settings.

Keywords: Cancer, Quality of Life, Patient Reported Outcomes, Health Services Research

\section{Background}

Patient reported outcome (PRO) measures include health status assessments and measures for healthrelated quality-of-life (HRQOL), symptom reporting, satisfaction with care, treatment satisfaction, economic impact, and specific dimensions of patient experience such as depression and anxiety [1]. The USA Food and Drug Agency (FDA) adopts a much broader definition [2] as "A PRO is any report coming directly from patients

\footnotetext{
* Correspondence: jackchen@unsw.edu.au

${ }^{1}$ The Simpson Centre for Health Services Research, South Western Sydney Clinical School, University of New South Wales, Liverpool 2170NSW, Australia ${ }^{2}$ Australian Institute of Health Innovation, Level 1, AGSM Building, University of New South Wales, Randwick 2052, Australia
}

about a health condition and its treatment", meaning that PROs capture patients' perspectives about how illness or new therapies impact on their general well-being . There is a growing interest from clinicians, researchers, industry and policy-makers in routinely collecting PROs to facilitate timely, patient-centred and evidence-based care. For example, the National Health Service (NHS) of the UK has been implementing a world-leading initiative for the routine collection of PROs that firstly included a few selected elective surgeries (e.g. unilateral hip replacements, unilateral knee replacements, groin hernia surgery or varicose vein surgery) [3] but are soon expanding to many other conditions such as mastectomy 
and breast cancer, among others. In the USA, the Patient-Reported Outcomes Measurement Information System (PROMIS), a National Institutes of Health funded initiative starting in 2004, is providing a publicly available web-based resource that can be used to measure key health symptoms and HRQOL [4]. The traditional paper-based PROs instruments are limited by its lack of flexibility, language and literacy requirement, $[5,6]$ possible inappropriateness towards minority groups, $[7,8]$ lack of timeliness (in generating instantaneous clinical meaningful interpretations) [9] and inability to adopt state-of-the-art measurement science such as Item Response Theory (IRT) and Computer Adapted Test (CAT) technique [10]. To overcome the difficulty of integrating the administration and analysis of PRO instruments into clinical practice, researchers are developing and validating alternatives to traditional paperbased instruments such as office-based touch-screen computers, [11-13] telephone-based interactive voiceresponse (IVR) systems, [14-16] hand-held computers, $[17,18]$ mobile phones, [19-21] and more recently, the Internet [22-24]. Some rationales [25-28] put forward for measuring PROs in a cancer setting include, but not limited to: 1) better communication and shared decision making by patients and providers; 2) assessing the health status of patients entering therapy and identifying treatable problems; 3) determining the degree and sources of the patient's decreased ability to function; 4) distinguishing between types of problems, including physical, emotional, and social; 5) detecting adverse effects of therapy; 6) monitoring the effects of disease progression and response to therapy; 7) informing decisions about changing treatment plans, and 8) predicting the course of disease and outcomes of care.

However, despite growing interest and urges by the leading experts for applying routinely collected PROs for all cancer patients, there has not been an updated comprehensive review of the evidence regarding the impact of adopting such a strategy on patients, services providers and organisations. The most recent review focused only on clinical trial design [26] studies of cancer patients, and only assessed a limited number of outcomes. The current project aims to provide the much needed comprehensive review update, including all relevant quantitative studies investigating the effectiveness of routine $\mathrm{PRO}$ collection in cancer patients. The review research questions were:

1. What are the impacts of composite measures of PROs collected on cancer patients during treatment with regards to:

a) Provider behaviour for improving care delivered;

b) Organisational changes within health care settings for improving processes and models of care (e.g. targeting and tailoring care); c) Improving clinical outcomes for patients; and

d) Improving patient experience of care (e.g. self-care).

2. What mechanisms are involved in the link between PROs and the impacts identified in 1(a)?

3. What factors moderate the extent of the impacts identified in $1(\mathrm{a})$ ?

\section{Methods}

\section{Existing systematic reviews and rationale for the current} review

In order to develop an efficient search and review strategy, over 200 existing reviews on the same or similar topics were firstly systematically examined (identified in a broad search covering PROs and quality of life measures between January 2000 and October 2011). Three reviews [26-28] were identified as the baseline reviews for this project and their review strategies were carefully examined in aspects such as the aim and scope, time span, search strategy and search terms used, articles included in each review, and conclusions drawn. A table summarising the three systematic reviews is presented in Table 1.

\section{Review search strategy}

Analysing the results of above three systematic reviews demonstrates the importance of search strategies in determining what literature will be included in the study, which in turn, may influence what conclusions will be derived. Valderas et al.'s (2008) [27] review excluded three out of the five clinical trials on cancer patients that were included in Marshall et al.'s (2006) [28] review. Lucket et al.'s (2009) review [26] excluded one article (Taenzer et al. (2000), [33] a before-after study) from Marshall et al.'s review [27]. A mixed methodology search was developed in order to maximise the identification of recent literature in a short period of time. The search was conducted in six different ways as follows:

1. A text-based search strategy was developed based on previous reviews. To elicit previous reviews, a search was conducted for the text terms 'patient reported outcome", 'self-reported,' 'self-assessed' anywhere in title, abstract and key words, combined with 'quality of life,' 'symptom,' 'functional status,' 'health status', 'patient satisfaction,' 'unmet need"' anywhere in title, abstract and key words. For original articles, a search was conducted using the same strategy as above but restricted to those with 'neoplasm' or 'cancer' in the key words. The search results were restricted to between January 2000 and October 2011 (full search strategy is listed in Additional file 1: Appendix 1).

2. All reviews were evaluated (over 200 in total on various topics but not limited to only cancer 
Table 1 A comparison of three baseline reviews

\begin{tabular}{|c|c|c|c|c|c|}
\hline $\begin{array}{l}\text { First author, } \\
\text { year }\end{array}$ & Aim and review scope & Time span and the search strategy & Search Terms & $\begin{array}{l}\text { Articles included in } \\
\text { the review }\end{array}$ & Major conclusions \\
\hline $\begin{array}{l}\text { Luckett et al. } \\
2009 \text { [26] }\end{array}$ & $\begin{array}{l}\text { To identify future strategies for (1) } \\
\text { interventions to impact patient } \\
\text { outcomes; and (2) trials to identify } \\
\text { treatment effects. }\end{array}$ & $\begin{array}{l}\text { MEDLINE and PsycINFO were } \\
\text { systematically searched to identify } \\
\text { reports of relevant randomised } \\
\text { controlled trials. The time span was } \\
\text { between } 2006 \text { and } 1 \text { August } 2008 \text {. } \\
\text { Four cancer trials were cited in a } \\
\text { previous review (Valderas et al. 2008) } \\
\text { [27]. }\end{array}$ & $\begin{array}{l}\text { 1. Examined the citations of the four } \\
\text { trials 2. Adopted the strategy used by } \\
\text { Valderas et al. [27] and Espallargues } \\
\text { et al. [29] which involved searching for } \\
\text { the terms 'health status', 'functional } \\
\text { status' or 'quality of life' and 'clinical } \\
\text { practice', 'clinical setting', 'practice } \\
\text { setting', 'medical practice' or 'medical } \\
\text { consultation' anywhere in the title, } \\
\text { abstract or keywords. Results were } \\
\text { limited by publication date (2006- } \\
\text { 2008) and the MeSH or keyword } \\
\text { neoplasm. }\end{array}$ & 6 RCTs & $\begin{array}{l}\text { Future interventions should motivate } \\
\text { and equip health professionals to use } \\
\text { PROs data in managing patients, } \\
\text { training patients in self-efficacy, using } \\
\text { more specific PROs in clinics, } \\
\text { improving the interpretability of } \\
\text { feedback for both medical staff and } \\
\text { patients, and monitoring the use of } \\
\text { PROs to intervene when problems } \\
\text { arise. Future trials should use a cluster } \\
\text { randomised design to control for } \\
\text { contamination and enable systems- } \\
\text { based interventions. }\end{array}$ \\
\hline $\begin{array}{l}\text { Valderas et al. } \\
2008 \text { [27] }\end{array}$ & $\begin{array}{l}\text { To summarize the best evidence } \\
\text { regarding the impact of providing } \\
\text { patient reported outcomes (PRO) } \\
\text { information to health care } \\
\text { professionals in daily clinical practice. }\end{array}$ & $\begin{array}{l}\text { Systematic review of randomised clinical } \\
\text { trials (Medline, Cochrane Library); } \\
\text { reference lists of previous systematic } \\
\text { reviews; and requests to authors and } \\
\text { experts in the field. Time span: Articles } \\
\text { published between } 1978 \text { and } 2007 .\end{array}$ & $\begin{array}{l}\text { No exact search terms provided but } \\
\text { indicated available from the author } \\
\text { upon request. }\end{array}$ & $\begin{array}{l}34 \text { articles } \\
\text { corresponding to } 28 \\
\text { original studies; only } 2 \\
\text { (not 4) as mentioned in } \\
\text { the above review, are in } \\
\text { an oncologic setting. }\end{array}$ & $\begin{array}{l}\text { Methodological concerns limit the } \\
\text { strength of inference regarding the } \\
\text { impact of providing PROs } \\
\text { information to clinicians. Results } \\
\text { suggest great heterogeneity of } \\
\text { impact; contexts and interventions } \\
\text { that will yield important benefits } \\
\text { remain to be clearly defined. }\end{array}$ \\
\hline $\begin{array}{l}\text { Marshall et al. } \\
2006[28]\end{array}$ & $\begin{array}{l}\text { To synthesize the evidence for using } \\
\text { publically reported performance data } \\
\text { to improve quality. Only articles that } \\
\text { provided empirical evidence on the } \\
\text { impact of public reporting on } \\
\text { outcomes (effectiveness, patient } \\
\text { safety, and patient-centeredness) and } \\
\text { unintended consequences, as well as } \\
\text { selection and quality improvement } \\
\text { activity were included. }\end{array}$ & $\begin{array}{l}\text { Webspirs Medline was searched for } \\
\text { the years from January } 1976 \text { to } \\
\text { November 2004. Reference lists of } \\
\text { included studies and appropriate } \\
\text { reviews (Greenhalgh \& Meadows } 1999 \\
\text { [30]; Espallargues et al. } 2000 \text { [29]; } \\
\text { Gilbody et al. } 2003 \text { [31]) were also } \\
\text { searched for relevant articles. Finally, } \\
\text { PubMed's 'related articles' feature was } \\
\text { used with several background and } \\
\text { included articles (Drury et al. } 2000 \text { [32]; } \\
\text { Velikova et al. } 2004 \text { [13]) to identify } \\
\text { publications with a high proportion of } \\
\text { similar text in the title and abstract. }\end{array}$ & $\begin{array}{l}\text { Terms used in relation to patient- } \\
\text { reported outcome measures (for } \\
\text { example, 'self report* near2 } \\
\text { measure*') joined with an 'and' } \\
\text { command to terms related to routine }_{\text {practice outcomes (for example, }} \\
\text { 'improve* near detect*) or patient } \\
\text { involvement in the health care } \\
\text { process (such as 'patient* near } \\
\text { provider* near interaction*'). }\end{array}$ & $\begin{array}{l}40 \text { articles included in } \\
\text { the review including } 5 \\
\text { publications from an } \\
\text { oncologic setting. }\end{array}$ & $\begin{array}{l}\text { The pattern of results suggests a } \\
\text { general lack of clarity in the field, } \\
\text { especially regarding appropriate goals } \\
\text { for PROs and the mechanisms by } \\
\text { which they might achieve them. To } \\
\text { fully evaluate their role in routine } \\
\text { practice, studies need to use PROs that } \\
\text { capture issues of importance to } \\
\text { patients and to measure impacts } \\
\text { relating to the patient-provider } \\
\text { relationship and patient contributions } \\
\text { to their well-being. Until studies } \\
\text { evaluate PROs as a means to facilitate } \\
\text { patient-centred care, their full potential } \\
\text { in clinical practice will remain unknown. }\end{array}$ \\
\hline
\end{tabular}


patients) with three baseline reviews used as the starting point for our top-down and bottom-up search strategy. We chose the three baseline reviews because that: 1 ) they are all systematic reviews that could be helpful in forming the structure or strategy of the current review (but not necessarily restricted to cancer patients); and 2) they were published after 2005.

3. All articles were examined if they cited the 7 key randomised controlled trials [33-39] listed in the above reviews (bottom-up approach). References were also sought from the most recently published trials, editorials, and commentaries (a top-down approach). The powerful citation tracking feature of Scopus $^{\text {Tx }}$ made this strategy feasible.

4. Simplified text terms (i.e. patient reported outcome, PRO, PROM, Quality of life, QOL) were used to conduct a web search for identifying grey literature.

5. Leading researchers and experts in the field (elicited through the advice of Cancer Institute NSW (CINSW), editorials, review articles and most cited articles) were purposefully searched in order to analyse the references and citations in their publications.

6. Some key cancer centres' websites were also searched in order to get more detailed information.

The search was limited to the Scopus ${ }^{\text {Tum }}$ database as it is the largest abstract and citation database of peer-reviewed literature and quality web sources including $100 \%$ coverage of Medline titles and EBASE. It also tracks, analyses and visualises publication results, which is well suited to our top-down and bottom-up search strategy.

\section{Aim, study selection and endpoints of the review}

In this review, the aim was to synthesize the evidence in relation to the impact of routinely collected PROs on patients, providers, and health organisations. The frameworks proposed by Greenhalgh and colleagues [25] and by Abernethy and colleagues [40] were adopted to guide our evaluation of the existing literature. Greenhalgh et al. [25] proposed a framework (Figure 1) that depicts mechanisms between the routine collection of PROs and changes in patient outcomes. The authors suggest that the multilayer mediators (i.e. changes to doctor-patient communication, monitoring treatment responses, detecting unrecognised problems, changes to patient health behaviour, changes to clinicians' management plans, and improved patient satisfaction) have complex relationships among them. The studies that revealed these complex relationships may assist in understanding whether and how the underlying mechanisms of routinely collected PROs work to improve the intended outcomes.

Recently, Abernethy and colleagues [40] have argued that the routine collection of PROs has the capacity to impact not only at the patient-level, but by addressing the logistics of data linkage, and could ensure that the system will grow to accommodate other clinical- and health system-level issues; for example, evaluating comparative effectiveness of treatments, monitoring quality of care, and translating basic science findings into clinical practice (Figure 2). The integration of data systems will fuel rapid learning cancer care at the national and societal levels (see Figure 2a and b), making many types of research and system learning possible across institutions and health sectors. The benefits and implications of such a rapid learning health care system may include, but is not limited to, strong and effective quality improvement (QI), increased transparency, accountability, public reporting, better health system performance (monitoring, planning, financing, evaluating, responding) and better quality of care.

Combining both frameworks, a list of outcome indicators was developed (Table 2) against which each eligible study was assessed. To include not only the doctors' experience with patients after collecting PROs, but also the experience of other health services providers (i.e. nurses, allied health workers), the term 'Patient-provider communication' was used instead of 'doctor patient communication' as proposed by Greenhalgh et al. [25]. In order to answer review questions $2 \& 3$ for the studies included, all possible explicit mediation effects were reviewed through examining if a path-analysis or a mediation-analysis by multiple, staged regression approach was presented in the paper. To examine potential moderating effect, each study was examined to determine if it explicitly tested the interaction effect/moderating effect, or inexplicitly conducted subgroup analysis. Significant possible mediating or moderating effect results were indicated as part of review endpoints in Table 3. Inferences made and the discussion were based on these results.

\section{Inclusion and exclusion criteria}

The inclusion criteria were: 1) substantial content in presenting empirical evidence on the impact of routinely collected PROs on at least one of the outcomes listed in Table 2; 2) adult cancer patients; 3) conducted in an oncologic setting including inpatient, outpatient and outreach services; and 4) studies using a composite PRO. We defined a composite PRO as those PROs are often based on a welldeveloped instrument and with an aim for measuring a substantial aspect of patient conditions (or treatment) with at least 4 items. To reflect the demanding and complex nature of evaluating the impact of routine collected PROs, eligible studies included a variety of designs including, but not limited to, randomised controlled trials (RCTs), controlled before-after trials (CBA) and interrupted time series (ITS). ITS designs have a longitudinal character, with repeated measurements and at least three data points before and after the intervention point. Surveys and clinical audits 


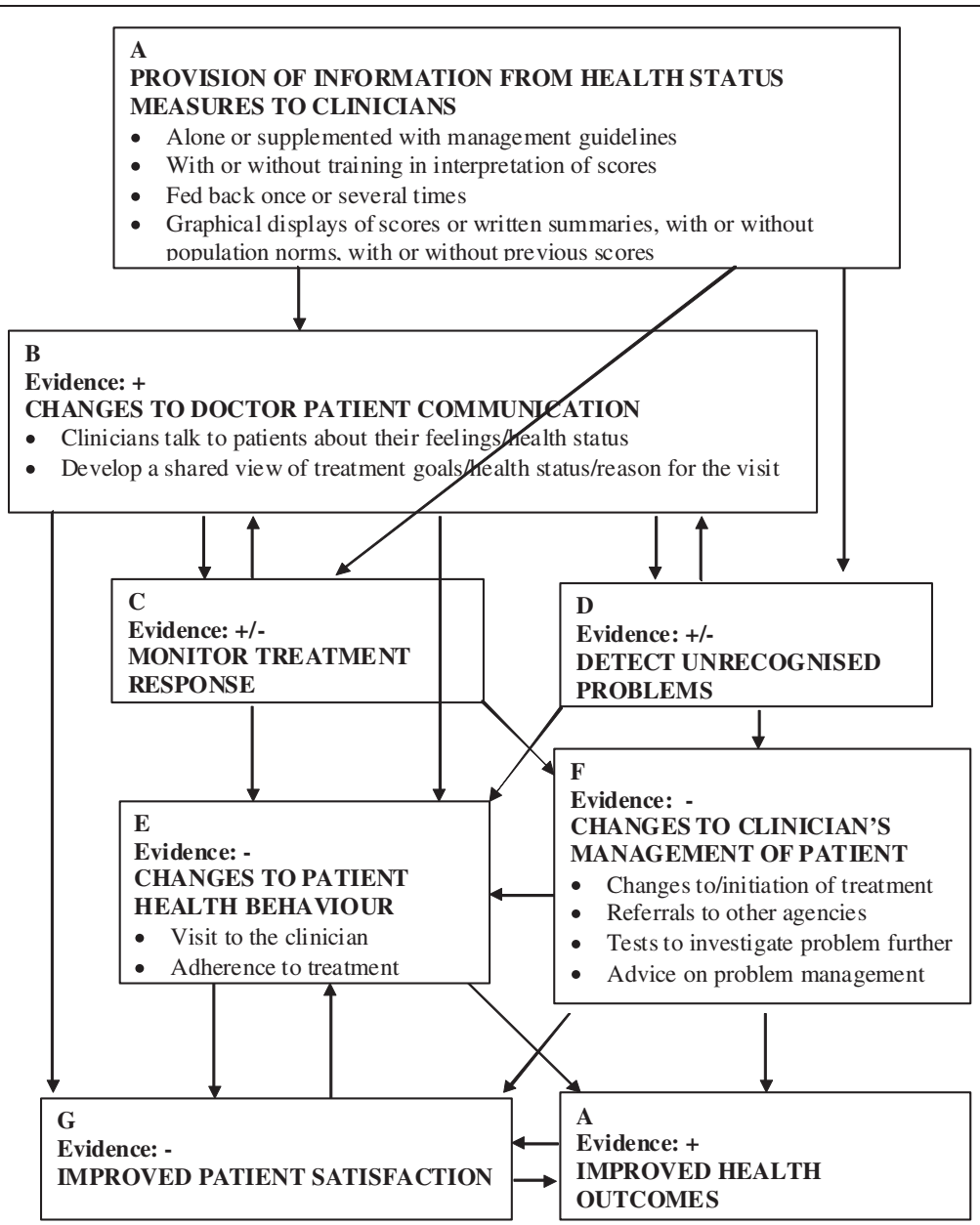

Figure $1 \mathrm{~A}$ hypothetical framework to understand the impact of routinely collected PROs on patient health outcomes (adopted from Greenhalgh et al. (2005) [25] with permission).

were also included if the studies provided quantitative results relevant to the listed outcomes.

Studies were excluded if they were non-English language articles, opinion and theoretical articles, historical descriptions, review articles, feasibility studies of some PROs collection devices, studies investigating child cancer patients or qualitative studies with no substantial quantitative results on the review endpoints.

\section{Data extraction and quality assessment}

Electronic search results were downloaded into EndNote bibliographic software. Two reviewers independently

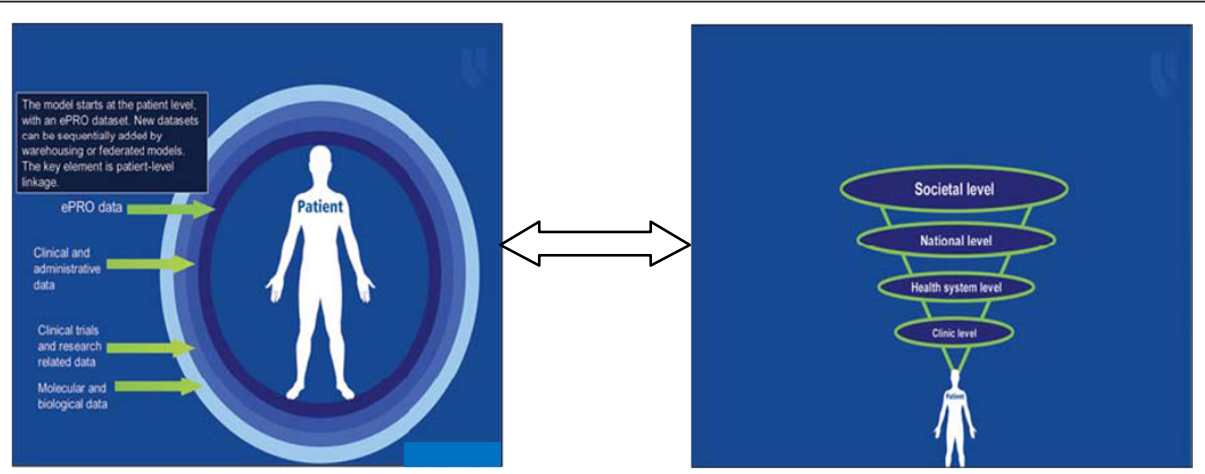

Figure 2 (a) A data linkage framework (b): A learning health care system. Note: Figures 2: adopted from Aberthnethy et al. (2010) [40] with permission. 


\begin{tabular}{|c|c|}
\hline Number & Outcomes \\
\hline 1 & Patient-provider communication \\
\hline 2 & Monitor treatment response \\
\hline 3 & Detect unrecognised problems \\
\hline 4 & Changes to patient health behaviour \\
\hline 5 & Changes to patient management \\
\hline 6 & Improved patient satisfaction \\
\hline 7 & Improved health outcomes \\
\hline 8 & Strong \& effective quality improvement \\
\hline 9 & Increased transparency, accountability, public reporting \\
\hline 10 & $\begin{array}{l}\text { Better system performance (monitoring, planning, financing; } \\
\text { evaluating, responding) }\end{array}$ \\
\hline 11 & $\begin{array}{l}\text { Mediating variables on the effect (both at individual and } \\
\text { organisation level) }\end{array}$ \\
\hline 12 & Possible subgroup effects \\
\hline
\end{tabular}

Note: Both 11 \& 12 are combined in the summarising tables as few studies have explored such issues.

(JC, LO) screened all titles and abstracts of citations identified by the electronic search, applied the selection criteria to potentially relevant papers, and extracted data from included studies using a standardised form. Any disagreements concerning studies to be included were resolved by consensus.

All studies were classified into two domains. Domain 1 correlated sample characteristics with population wide characteristics, and Domain 2 focused on study design. The data extraction form was adapted from other review studies using the outcome measures discussed above (see Table 4). For each eligible study, a list was made including the leading author, country and jurisdiction, design, sample, outcome measures, the PROs used, times of feedback and intervention, members of medical teams given feedback, management plans offered to teams, and training (see Table 5). All qualifying studies were listed chronologically with the outcome indicators (see Table 3).

In Domain 1, the routinely collected PROs in particular participants or samples was classified as rated on a 4point scale representing how closely the participants or samples overlapped with the characteristics and needs of the intended study populations ( 1 star=very weakly related to 4 stars =very strong related). For example, for a study conducted in the US on a sample of lung cancer patients, the degree of overlap of the study sample with the characteristics of lung cancer patients in the US overall was assessed by considering the study setting, sample size and sampling frame, response rate, loss-to-follow-up, and characteristics of the study sample. In Domain 2, study design was classified and rated on 4 categories with 1 star indicating the weakest design and 4 stars indicating the strongest design. Four stars indicated a randomised trial or experimental study; 3 stars indicated a controlled trial, pre-post trial with control (controlled before-after trial), time series, or observational cohort with multivariable adjustment; 2 stars indicated a pre-post trial without control, observational cohort study without multivariable adjustment, cross-sectional study without multivariable adjustment, analysis of time trends without control, or well-designed qualitative study; and 1 star indicated a case series, other qualitative study, or survey (descriptive) study.

Revised appraisal criteria were adapted from the guidelines on the assessment of quality improvement interventions [58,59]. A global rating was also created using the Grading of Recommendations, Assessment, Development, and Evaluation (GRADE) system [60]. The British Medical Journal has recommended the GRADE system since 2006 for grading evidence when submitting a clinical guidelines article. It has multiple advantages and is useful for systematic reviews and health technology assessments, as well as for evaluating research on clinical guidelines. The global rating created in the current study was based on the integration of the Domain 1 and Domain 2 ratings, as well as the intervention fidelity (the degree of success of the interventional strategy, the patients' and providers' adherence to the intervention strategy), dose-response gradient, precision and validity of outcomes (potential confounding factors and biases), and uncertainty of the direction of results. The global rating was divided into three categories; indication that the study should carry great ( 3 checks), moderate (2 checks), or little (1 check) weight when considering the strength of evidence (see Table 4). Any experimental research that is reported in the manuscript was performed with the approval of an appropriate ethics committee.

No attempt was made to quantitatively synthesize the results as the data were too heterogeneous to support pooling.

\section{Results}

The multi-method search strategy yielded 27 publications that were eligible to be included in the review - a significant increase compared with most of the recent reviews. The detailed flowchart of the search strategies and its relative results was presented (Figure 3). The results and conclusions drawn were based on the 27 studies included in the analyses despite the large number of full-text articles extracted.

Of the 27 publications, 16 were identified as randomised controlled trials, 2 as before-after studies and 9 observational studies with 11 studies published before 2009. The characteristics and quality of the studies are presented in Table 5 with their impact on outcome indicators presented in Table 3. As Trowbridge et al. (1997) was the only article in the 1990s included in two of the previous 
Table 3 The characteristics of design and study quality

\begin{tabular}{|c|c|c|c|c|c|c|c|c|c|c|c|c|}
\hline Reference & $\begin{array}{l}\text { Country / } \\
\text { Jurisdiction }\end{array}$ & Design & $\begin{array}{l}\text { Sample / } \\
\text { Population }\end{array}$ & $\begin{array}{l}\text { Outcome } \\
\text { measures }\end{array}$ & PROS used & $\begin{array}{l}\text { Intervention / } \\
\text { Number of } \\
\text { times feedback }\end{array}$ & $\begin{array}{l}\text { Members of } \\
\text { medical team } \\
\text { given feedback }\end{array}$ & $\begin{array}{l}\text { Management } \\
\text { plan offered } \\
\text { to team }\end{array}$ & $\begin{array}{l}\text { Training to } \\
\text { staff }\end{array}$ & $\begin{array}{l}\text { Domain } \\
1\end{array}$ & $\begin{array}{l}\text { Domain } \\
2\end{array}$ & $\begin{array}{l}\text { Global } \\
\text { Rating }\end{array}$ \\
\hline $\begin{array}{l}\text { Trowbridge } \\
\text { et al. (1997) } \\
\text { [39] }\end{array}$ & $\begin{array}{l}\text { USA (Central } \\
\text { Indiana } \\
\text { Community } \\
\text { Cancer Centres, } \\
\text { Indianapolis) }\end{array}$ & $\begin{array}{l}\text { RCT: } \\
\text { Intervention / } \\
\text { Control }\end{array}$ & $\begin{array}{l}320 \text { cancer } \\
\text { outpatients, } \\
13 \text { oncologists } \\
\text { and } 23 \text { clinics }\end{array}$ & $\begin{array}{l}\text { Pain } \\
\text { Management } \\
\text { Index(ref); pain } \\
\text { medication level } \\
\text { (0-3) minus pain } \\
\text { level: Patient } \\
\text { assessment of } \\
\text { pain, pain } \\
\text { regiments and } \\
\text { relief received } \\
\text { Patterns of } \\
\text { analgesic } \\
\text { prescription }\end{array}$ & $\begin{array}{l}\text { Estimates of } \\
\text { average and } \\
\text { worst pain over } \\
\text { the previous } 7 \\
\text { days, satisfaction } \\
\text { with current pain } \\
\text { regimens and } \\
\text { degree of relief } \\
\text { received }\end{array}$ & One & $\begin{array}{l}\text { Doctors } \\
\text { only (12) }\end{array}$ & No & No & ** & $* * *$ & $\sqrt{ } \sqrt{ }$ \\
\hline $\begin{array}{l}\text { Tazenzer } \\
\text { et al. (2000) } \\
\text { [33] }\end{array}$ & $\begin{array}{l}\text { Canada (Tom } \\
\text { Baker Cancer } \\
\text { Centre, Calgary, } \\
\text { Alberta) }\end{array}$ & $\begin{array}{l}\text { Before-after } \\
\text { trial: usual } \\
\text { care group / } \\
\text { Intervention } \\
\text { group with } \\
\text { before as } \\
\text { control }\end{array}$ & $\begin{array}{l}53 \text { lung cancer } \\
\text { patients } \\
\text { attending an } \\
\text { outpatient lung } \\
\text { cancer clinic }\end{array}$ & $\begin{array}{l}\text { EORTIC QLQ-C30 } \\
\text { 11-item Patient } \\
\text { Satisfaction } \\
\text { Questionnaire } \\
\text { (PDIS) ( adapted } \\
\text { through Falvo } \\
\text { and Smith,1983) } \\
\text { Exit Interview } \\
\text { (patient's } \\
\text { perception if QL } \\
\text { issues had been } \\
\text { addressed during } \\
\text { the visit) Medical } \\
\text { Record Audit on } \\
\text { patients' care plan }\end{array}$ & $\begin{array}{l}\text { EORTC QLQ-C30 } \\
\text { (on a PC) }\end{array}$ & Once & $\begin{array}{l}\text { Doctors and } \\
\text { nurses }\end{array}$ & No & $\begin{array}{l}\text { Ground round } \\
\text { introduction } \\
\text { and training }\end{array}$ & * & $* * *$ & $\sqrt{ } \sqrt{ }$ \\
\hline $\begin{array}{l}\text { McLachlan } \\
\text { et al. (2001) } \\
\text { [38] }\end{array}$ & $\begin{array}{l}\text { Australia (Peter } \\
\text { MacCallum } \\
\text { Cancer Centre, } \\
\text { Melbourne) }\end{array}$ & $\begin{array}{l}\text { RCT: } \\
\text { Intervention / } \\
\text { control (ratio: 2:1) }\end{array}$ & $\begin{array}{l}450 \text { cancer } \\
\text { patients } \\
\text { attending } \\
\text { ambulatory } \\
\text { clinics }\end{array}$ & $\begin{array}{l}\text { Patient HRQoL } \\
\text { (EORTC QLQ-C30) } \\
\text { 32-item Patient } \\
\text { needs (Cancer } \\
\text { Needs } \\
\text { Questionnaire } \\
\text { Short Form [CNQ] } \\
\text { Patient distress } \\
\text { (Beck Depression } \\
\text { Inventory (BDI) } \\
\text { Patient satisfaction } \\
\text { (in 6-month) } \\
\text { Services provided } \\
\text { for those } \\
\text { identified as } \\
\text { required by } \\
\text { coordination } \\
\text { nurse }\end{array}$ & $\begin{array}{l}\text { EORTC QLQ-C30, } \\
\text { CNQ, BDI (through } \\
\text { a touch-screen PC) }\end{array}$ & One & $\begin{array}{l}\text { Doctor and } \\
\text { coordination } \\
\text { nurse (numbers } \\
\text { not reporter) }\end{array}$ & $\begin{array}{l}\text { Individualised } \\
\text { plan } \\
\text { developed by } \\
\text { coordination } \\
\text { nurse in } \\
\text { accordance } \\
\text { with generic } \\
\text { psychosocial } \\
\text { guidelines }\end{array}$ & No & $* *$ & $* * *$ & $\sqrt{ } \sqrt{ }$ \\
\hline
\end{tabular}


Table 3 The characteristics of design and study quality (Continued)

\begin{tabular}{|c|c|c|c|c|c|c|c|c|c|c|c|c|}
\hline $\begin{array}{l}\text { Detmar et al. } \\
\text { (2002) [37] }\end{array}$ & $\begin{array}{l}\text { Netherland } \\
\text { (Netherlands } \\
\text { Cancer Institute, } \\
\text { Amsterdam) }\end{array}$ & $\begin{array}{l}\text { RCT: } \\
\text { (Cross-over } \\
\text { design) } \\
\text { Intervention/ } \\
\text { control }\end{array}$ & $\begin{array}{l}214 \text { palliative } \\
\text { cancer patients } \\
\text { in a outpatient } \\
\text { clinic of a } \\
\text { cancer hospital }\end{array}$ & $\begin{array}{l}\text { Patient-doctor } \\
\text { communication } \\
\text { Doctor's } \\
\text { awareness of } \\
\text { patient HRQoL } \\
\text { Patient } \\
\text { management } \\
\text { Patients'/doctors' } \\
\text { satisfaction } \\
\text { Patient HRQoL } \\
\text { (SF-36) Patients'/ } \\
\text { doctors' } \\
\text { evaluation of } \\
\text { intervention }\end{array}$ & EORTC QLQ-30 & Three & Doctors $(n=10)$ & No & $\begin{array}{l}\text { Doctors given } \\
30 \text {-mins } \\
\text { training and } \\
\text { patient } \\
\text { mailed a } \\
\text { leaflet }\end{array}$ & $* * *$ & $* * * *$ & $\sqrt{ } \sqrt{ } \sqrt{ }$ \\
\hline $\begin{array}{l}\text { Mooney } \\
\text { et al. (2002) } \\
\text { [16] }\end{array}$ & $\begin{array}{l}\text { USA (University } \\
\text { of Utah, Salt } \\
\text { Lake City, Utah) }\end{array}$ & $\begin{array}{l}\text { A pilot } \\
\text { Prospective } \\
\text { study over a } \\
\text { month period } \\
\text { with daily } \\
\text { measures }\end{array}$ & $\begin{array}{l}27 \text { patients } \\
\text { receiving } \\
\text { cancer } \\
\text { chemotherapy } \\
\text { at a cancer } \\
\text { centre } \\
\text { outpatient clinic }\end{array}$ & $\begin{array}{l}\text { Telephone-Linked } \\
\text { Care system for } \\
\text { Chemotherapy } \\
\text { (TLC-Chemo } \\
\text { Alert) Seven } \\
\text { symptoms } \\
\text { (nausea and } \\
\text { vomiting, fatigue, } \\
\text { trouble sleeping, } \\
\text { sore mouth, fever, } \\
\text { feeling blue, } \\
\text { feeling anxious) } \\
\text { Exit interview }\end{array}$ & TLC-Chemo Alert & $\begin{array}{l}\text { Patients asked } \\
\text { to report daily } \\
\text { during the } \\
\text { cycle and the } \\
\text { alerts were } \\
\text { sent to } \\
\text { providers }\end{array}$ & Doctors $(n=2)$ & Yes & $\begin{array}{l}\text { Patients } \\
\text { trained (10 } \\
\text { minutes TLC } \\
\text { orientation) }\end{array}$ & $* *$ & $* *$ & $\sqrt{ }$ \\
\hline $\begin{array}{l}\text { Velikova } \\
\text { et al. (2004) } \\
\text { [36] }\end{array}$ & $\begin{array}{l}\text { UK (Cancer } \\
\text { Research UK } \\
\text { Clinical Centre } \\
\text { - Leeds) }\end{array}$ & $\begin{array}{l}\text { RCT: } \\
\text { Intervention } \\
\text { /control- } \\
\text { attention/control } \\
\text { in a ratio of 2:1:1 }\end{array}$ & $\begin{array}{l}286 \text { cancer } \\
\text { outpatients } \\
\text { attending a } \\
\text { large cancer } \\
\text { centre of a } \\
\text { teaching } \\
\text { hospital }\end{array}$ & $\begin{array}{l}\text { Patient HRQoL } \\
\text { (FACT-G) } \\
\text { Discussion of } \\
\text { HRQoL issues in } \\
\text { consultation } \\
\text { Medical actions } \\
\text { (decisions on } \\
\text { cancer treatment, } \\
\text { symptomatic/ } \\
\text { supportive } \\
\text { treatment, } \\
\text { investigations and } \\
\text { referrals) Non- } \\
\text { medical actions } \\
\text { (advice on lifestyle, } \\
\text { copying and } \\
\text { reassurance) } \\
\text { Physician checklist } \\
\text { assessing the } \\
\text { clinical usefulness } \\
\text { of PROM data }\end{array}$ & $\begin{array}{l}\text { EORTC QOQ-C30 } \\
\text { Hospital Anxiety } \\
\text { and Depression } \\
\text { Scale (HADS) }\end{array}$ & $\begin{array}{l}\text { Regular clinic } \\
\text { visit over an } \\
\text { average of } 6 \\
\text { months }\end{array}$ & Doctor $(n=28)$ & No & $\begin{array}{l}\text { One to one } \\
\text { training and } \\
\text { manual } \\
\text { provided }\end{array}$ & $* * *$ & $* * * *$ & $\sqrt{ } \sqrt{ } \sqrt{ }$ \\
\hline
\end{tabular}


Table 3 The characteristics of design and study quality (Continued)

\begin{tabular}{|c|c|c|c|c|c|c|c|c|c|c|c|c|}
\hline $\begin{array}{l}\text { Basch et al. } \\
\text { (2005) [41] }\end{array}$ & $\begin{array}{l}\text { USA (Memorial } \\
\text { Sloan-Kettering } \\
\text { Cancer Center, } \\
\text { New York) }\end{array}$ & $\begin{array}{l}\text { Prospective pilot } \\
\text { study of patient } \\
\text { online self- } \\
\text { reporting of } \\
\text { toxicity } \\
\text { symptoms }\end{array}$ & $\begin{array}{l}80 \text { patients } \\
\text { diagnosed with } \\
\text { a gynaecologic } \\
\text { malignancy } \\
\text { starting a new } \\
\text { chemotherapy } \\
\text { regimen }\end{array}$ & $\begin{array}{l}\text { Pattern of use of } \\
\text { a Self-reported } \\
\text { online Symptom } \\
\text { Track and } \\
\text { Reporting (STAR) } \\
\text { system Patient } \\
\text { impression of } \\
\text { such system } \\
\text { based on an exit } \\
\text { questionnaire } \\
\text { survey Clinician } \\
\text { feedback } \\
\text { (through survey } \\
\text { and team } \\
\text { debriefing) }\end{array}$ & $\begin{array}{l}\text { Symptom Track } \\
\text { and Reporting } \\
\text { (STAR) based on } \\
\text { NCI CATAE system }\end{array}$ & $\begin{array}{l}\text { Any clinic visits } \\
\text { during 8-wk } \\
\text { study period } \\
\text { (mean=3, } \\
\text { range 1-6), } \\
\text { also possible } \\
\text { log in at home } \\
\text { during the } \\
\text { period }\end{array}$ & $\begin{array}{l}\text { Doctors and } \\
\text { study team } \\
\text { (n=unreported) }\end{array}$ & Yes & $\begin{array}{l}\text { Training } \\
\text { provide to } \\
\text { patients but } \\
\text { unreported } \\
\text { to staff }\end{array}$ & $* *$ & $* *$ & $\sqrt{ }$ \\
\hline $\begin{array}{l}\text { Boyes et al. } \\
\text { (2006) [35] }\end{array}$ & $\begin{array}{l}\text { Australia } \\
\text { (Centre for } \\
\text { Health Research } \\
\text { \& Psycho- } \\
\text { oncology, } \\
\text { University of } \\
\text { Newcastle) }\end{array}$ & $\begin{array}{l}\text { Pilot controlled } \\
\text { trial: Intervention } \\
\text { /control }\end{array}$ & $\begin{array}{l}80 \text { cancer } \\
\text { outpatients } \\
\text { attending one } \\
\text { cancer centre }\end{array}$ & $\begin{array}{l}\text { Patient symptoms } \\
\text { Patient anxiety/ } \\
\text { depression(HADS) } \\
\text { Patient needs } \\
\text { (Supportive Care } \\
\text { Needs Survey } \\
\text { [SCNS] } \\
\text { Acceptability of } \\
\text { intervention to } \\
\text { patient and } \\
\text { doctors }\end{array}$ & $\begin{array}{l}\text { Symptoms, } \\
\text { HADS SCNS }\end{array}$ & $\begin{array}{l}1^{\text {st }} \text { consultation } \\
-100 \% \text { patients: } \\
2^{\text {nd }}: 83 \% ; 3^{\text {rd: }} \\
71 \% ; 4^{\text {th }}: 60 \%\end{array}$ & Doctors $(n=4)$ & $\begin{array}{l}\text { List of patients } \\
\text { needs } \\
\text { accompanied } \\
\text { by } \\
\text { suggestions } \\
\text { for } \\
\text { appropriate } \\
\text { referral }\end{array}$ & None & $* *$ & $* * *$ & $\sqrt{ } \sqrt{ }$ \\
\hline $\begin{array}{l}\text { Hoeskstra } \\
\text { et al. (2006) } \\
{[42]}\end{array}$ & $\begin{array}{l}\text { Netherlands } \\
\text { (Academic } \\
\text { Medical } \\
\text { Centre, } \\
\text { University of } \\
\text { Amsterdam) }\end{array}$ & $\begin{array}{l}\text { RCT: Intervention } \\
\text { group with } \\
\text { symptom } \\
\text { monitoring / } \\
\text { control }\end{array}$ & $\begin{array}{l}146 \text { palliative } \\
\text { cancer patients } \\
\text { recruited } \\
\text { through two } \\
\text { hospitals and } \\
\text { local GPs }\end{array}$ & $\begin{array}{l}10 \text { symptoms } \\
\text { from the } \\
\text { Symptom } \\
\text { Monitor Severity } \\
\text { of the reported } \\
\text { symptom } \\
(0-10 \text { score) }\end{array}$ & $\begin{array}{l}\text { Symptom Monitor } \\
\text { Extensive } \\
\text { Questionnaire }\end{array}$ & $\begin{array}{l}\text { Weekly self- } \\
\text { assessed } \\
\text { Symptom } \\
\text { Monitor at } \\
\text { home; } \\
\text { Extensive } \\
\text { questionnaire } \\
\text { every 2-month }\end{array}$ & $\begin{array}{l}\text { GPs (98 times) } \\
\text { and medical } \\
\text { specialists } \\
\text { (96 times) }\end{array}$ & No & No & $* *$ & $* * *$ & $\sqrt{ } \sqrt{ }$ \\
\hline \multirow{8}{*}{$\begin{array}{l}\text { Korniblith } \\
\text { et al. (2006) } \\
\text { [43] }\end{array}$} & \multirow{8}{*}{$\begin{array}{l}\text { USA (Dana- } \\
\text { Farber Cancer } \\
\text { Institute, } \\
\text { Boston) }\end{array}$} & \multirow{8}{*}{$\begin{array}{l}\text { RCT : Telephone } \\
\text { Monitoring (TM) } \\
\text { versus TM } \\
\text { +Education } \\
\text { Material (EM) }\end{array}$} & \multirow{8}{*}{$\begin{array}{l}192 \text { cancer } \\
\text { patients with } \\
\text { advanced } \\
\text { disease and } \\
\text { receiving active } \\
\text { treatment }\end{array}$} & \multirow{8}{*}{$\begin{array}{l}\text { EORTC-QLQ-30 } \\
\text { HADS }\end{array}$} & EORTC-QLQ-30 & \multirow{8}{*}{$\begin{array}{l}\text { Once a month } \\
\text { over } 6 \text { months }\end{array}$} & \multirow{8}{*}{$\begin{array}{l}\text { Ontological } \\
\text { nurses }\end{array}$} & \multirow[t]{8}{*}{ Yes } & \multirow[t]{8}{*}{ Yes } & \multirow[t]{8}{*}{$* * *$} & \multirow[t]{8}{*}{$* * * *$} & \multirow[t]{8}{*}{$\sqrt{ } \sqrt{ }$} \\
\hline & & & & & HADS & & & & & & & \\
\hline & & & & & MOS-SS & & & & & & & \\
\hline & & & & & GDS (short form) & & & & & & & \\
\hline & & & & & QARSQ-PH & & & & & & & \\
\hline & & & & & UMPSI & & & & & & & \\
\hline & & & & & GSRE & & & & & & & \\
\hline & & & & & $\begin{array}{l}\text { Patient Satisfaction } \\
\text { with the Research } \\
\text { Program BOMC } \\
\text { test; }\end{array}$ & & & & & & & \\
\hline
\end{tabular}


Table 3 The characteristics of design and study quality (Continued)

\begin{tabular}{|c|c|c|c|c|c|c|c|c|c|c|c|c|}
\hline $\begin{array}{l}\text { Basch et al. } \\
\text { (2007) [44] }\end{array}$ & $\begin{array}{l}\text { USA (Memorial } \\
\text { Sloan-Kettering } \\
\text { Cancer Center, } \\
\text { New York) }\end{array}$ & $\begin{array}{l}\text { Prospective pilot } \\
\text { study of a } \\
\text { patient online } \\
\text { self-reporting of } \\
\text { toxicity } \\
\text { symptoms }\end{array}$ & $\begin{array}{l}107 \text { patients } \\
\text { diagnosed with } \\
\text { thoracic } \\
\text { gynaecologic } \\
\text { malignancy } \\
\text { starting a new } \\
\text { chemotherapy } \\
\text { regimen }\end{array}$ & $\begin{array}{l}\text { Feasibility/Pattern } \\
\text { of use of a Self- } \\
\text { reported online } \\
\text { Symptom Track } \\
\text { and Reporting } \\
\text { (STAR) system } \\
\text { Patient } \\
\text { satisfaction } \\
\text { survey } \\
\text { (an exit } \\
\text { questionnaire } \\
\text { survey) Nursing } \\
\text { survey (through } \\
\text { an exit survey) }\end{array}$ & $\begin{array}{l}\text { Symptom Track } \\
\text { and Reporting } \\
\text { (STAR) based on } \\
\text { NCI CATAE } \\
\text { system }\end{array}$ & $\begin{array}{l}\text { Any clinic visits } \\
\text { during } 42 \text {-wk } \\
\text { study period } \\
\text { (mean=12, } \\
\text { range 1-40), } \\
\text { also possible log } \\
\text { in at home } \\
\text { during the } \\
\text { period }\end{array}$ & $\begin{array}{l}\text { Nurses and } \\
\text { study team } \\
\text { (n=unreported) }\end{array}$ & No & $\begin{array}{l}\text { Training } \\
\text { provide to } \\
\text { patients but } \\
\text { unreported to } \\
\text { staff }\end{array}$ & $* *$ & $* *$ & $\sqrt{ }$ \\
\hline $\begin{array}{l}\text { Rosenbloom } \\
\text { et al. (2007) } \\
\text { [34] }\end{array}$ & $\begin{array}{l}\text { USA (Center on } \\
\text { Outcomes, } \\
\text { Research and } \\
\text { Education, } \\
\text { Evanston } \\
\text { Northwestern } \\
\text { Healthcare }\end{array}$ & $\begin{array}{l}\text { RCT: Structured } \\
\text { interview and } \\
\text { discussion / } \\
\text { assessment } \\
\text { control / } \\
\text { standard care }\end{array}$ & $\begin{array}{l}213 \text { patients } \\
\text { with advanced } \\
\text { breast, lung or } \\
\text { colorectal } \\
\text { cancer }\end{array}$ & $\begin{array}{l}\text { Patient HRQOL } \\
\text { (Functioning } \\
\text { Living Index- } \\
\text { Cancer [FLIC]) } \\
\text { Patient affect } \\
\text { (Brief Profile of } \\
\text { Mood States } \\
\text { [Brief POMS]) } \\
\text { Patient } \\
\text { satisfaction } \\
\text { [PSQ-III] Clinical } \\
\text { treatment } \\
\text { changes as } \\
\text { reported by nurse } \\
\text { (supportive care } \\
\text { changes, referrals, } \\
\text { 'other' clinical } \\
\text { changes and } \\
\text { changes in } \\
\text { standard dose of } \\
\text { chemotherapy as } \\
\text { a result of PROs) }\end{array}$ & $\begin{array}{l}\text { FACT-G and a } \\
\text { single item } \\
\text { asking patients } \\
\text { whether a } \\
\text { particular } \\
\text { symptom or } \\
\text { problem was } \\
\text { better than, } \\
\text { worse than, or } \\
\text { as expected }\end{array}$ & $\begin{array}{l}\text { Clinic visits at } \\
\text { baseline ,and 1, } \\
2,3 \text { and } 6 \\
\text { months }\end{array}$ & $\begin{array}{l}\text { Treating } \\
\text { nurses }(\mathrm{n}=\text { not } \\
\text { reported) }\end{array}$ & No & No & $* * *$ & $* * * *$ & $\sqrt{ } \sqrt{ } \sqrt{ }$ \\
\hline $\begin{array}{l}\text { Weaver et al. } \\
\text { (2007) [45] }\end{array}$ & $\begin{array}{l}\text { UK (Oxford } \\
\text { Radcliffe } \\
\text { Hospitals } \\
\text { NHS Trust) }\end{array}$ & $\begin{array}{l}\text { A pilot study of } \\
\text { novel mobile } \\
\text { phone } \\
\text { technology }\end{array}$ & $\begin{array}{l}6 \text { colon cancer } \\
\text { patients }\end{array}$ & $\begin{array}{l}\text { Questionnaire on } \\
\text { symptoms } \\
\text { derived from the } \\
\text { Common } \\
\text { Terminology } \\
\text { Criteria for } \\
\text { Adverse Events } \\
\text { (CTCAE) grading } \\
\text { system }\end{array}$ & $\begin{array}{l}\text { Questionnaire } \\
\text { derived from the } \\
\text { Common } \\
\text { Terminology } \\
\text { Criteria for } \\
\text { Adverse Events } \\
\text { (CTCAE) grading } \\
\text { system }\end{array}$ & $\begin{array}{l}\text { Twice daily } \\
\text { during the } \\
\text { chemotherapy } \\
\text { circle (one } \\
\text { morning, one } \\
\text { evening) }\end{array}$ & $\begin{array}{l}\text { Nurses } \\
\text { ( } n=\text { not } \\
\text { reported) }\end{array}$ & Yes & Yes & $* *$ & $* *$ & $\sqrt{ }$ \\
\hline $\begin{array}{l}\text { Butt et al. } \\
\text { (2008) [46] }\end{array}$ & $\begin{array}{l}\text { USA (Center } \\
\text { on Outcomes, } \\
\text { Research and }\end{array}$ & $\begin{array}{l}\text { Prospective } \\
\text { study to explore } \\
\text { the longitudinal }\end{array}$ & $\begin{array}{l}99 \text { cancer } \\
\text { patients with } \\
\text { solid tumor of }\end{array}$ & $\begin{array}{l}\text { FACT-G FACT- } \\
\text { Fatigue subscale } \\
\text { Brief Pain }\end{array}$ & $\begin{array}{l}\text { FACT-G FACT- } \\
\text { Fatigue subscale } \\
\text { Brief Pain }\end{array}$ & $\begin{array}{l}\text { Baseline, } 1 \\
\text { month and } 2\end{array}$ & $\begin{array}{l}\text { Doctors and } \\
\text { nurses }\end{array}$ & $?$ & $?$ & $* *$ & $* *$ & $\sqrt{ }$ \\
\hline
\end{tabular}


Table 3 The characteristics of design and study quality (Continued)

\begin{tabular}{|c|c|c|c|c|c|c|c|c|c|c|c|c|}
\hline & $\begin{array}{l}\text { Education } \\
\text { (CORE), Evanton } \\
\text { Northwestern } \\
\text { Healthcare) }\end{array}$ & $\begin{array}{l}\text { screening and } \\
\text { management of } \\
\text { fatigue, pain, and } \\
\text { emotional } \\
\text { distress }\end{array}$ & $\begin{array}{l}\text { lymphoma } \\
\text { undergoing } \\
\text { cancer } \\
\text { undergoing } \\
\text { cancer } \\
\text { treatment }\end{array}$ & $\begin{array}{l}\text { Inventory (BPI) } \\
\text { HADS Structured } \\
\text { interview with } \\
\text { patients on HRQL } \\
\text { and symptom } \\
\text { management }\end{array}$ & $\begin{array}{l}\text { Inventory (BPI) } \\
\text { HADS }\end{array}$ & $\begin{array}{l}\text { months after } \\
\text { the baseline }\end{array}$ & & & & & & \\
\hline $\begin{array}{l}\text { Given et al. } \\
\text { (2008) [47] }\end{array}$ & $\begin{array}{l}\text { USA( Michigan } \\
\text { State University) }\end{array}$ & $\begin{array}{l}\text { RCT: Nurse- } \\
\text { Administrated } \\
\text { Symptom } \\
\text { Management } \\
\text { (NASM) vs } \\
\text { Automated } \\
\text { Telephone } \\
\text { Symptom } \\
\text { Management } \\
\text { (ATSM) } \\
\text { intervention }\end{array}$ & $\begin{array}{l}129 \text { breast } \\
\text { cancer patients }\end{array}$ & $\begin{array}{l}\text { Outcomes } \\
\text { measured at } \\
10-16 \text { wks: } 15 \\
\text { symptoms } \\
\text { (0-10 scale) } \\
\text { Responses \& } \\
\text { Non-responses } \\
\text { of symptoms } \\
\text { Time to } \\
\text { response }\end{array}$ & $\begin{array}{l}15 \text { symptoms } \\
\text { (0-10 scale) } \\
\text { Severity of the } \\
\text { symptoms }\end{array}$ & $\begin{array}{l}6 \text { contacts or } \\
\text { self-reporting } \\
(1-4 w k, 6 w k, \\
8 w k)\end{array}$ & $\begin{array}{l}\text { Nurses or } \\
\text { ATSM system }\end{array}$ & Yes & Yes & $* *$ & $* * * *$ & $\sqrt{ } \sqrt{ } \sqrt{ }$ \\
\hline $\begin{array}{l}\text { Hilarius et al. } \\
\text { (2008) [48] }\end{array}$ & $\begin{array}{l}\text { Netherland } \\
\text { (Hospital } \\
\text { Pharmacy, Red } \\
\text { Cross Hospital, } \\
\text { Beverwijk) }\end{array}$ & $\begin{array}{l}\text { A sequential } \\
\text { cohort design } \\
\text { with repeated } \\
\text { measures to } \\
\text { evaluate the use } \\
\text { of HRQL } \\
\text { assessments in } \\
\text { daily clinical } \\
\text { oncology } \\
\text { nursing practice }\end{array}$ & $\begin{array}{l}10 \text { nurses and } \\
219 \text { patients } \\
\text { cancer patients } \\
\text { with either } \\
\text { adjuvant or } \\
\text { palliative } \\
\text { chemotherapy } \\
\text { in a community } \\
\text { hospital }\end{array}$ & $\begin{array}{l}\text { Dartmouth } \\
\text { Primary Care } \\
\text { Cooperative } \\
\text { Information } \\
\text { Functional Health } \\
\text { Assessment } \\
\text { (COOPcharts) } \\
\text { Patient } \\
\text { Management } \\
\text { extracted from } \\
\text { medical record } \\
\text { Patient } \\
\text { satisfaction } \\
\text { (an exit survey } \\
\text { based on PSQ, } \\
\text { Form II) Patients' } \\
\text { self-reported } \\
\text { HRQL (SF-36, } \\
\text { FACC-BCS, } \\
\text { FACT-C, FACT-L) } \\
\text { Nurse and patient } \\
\text { evaluation of the } \\
\text { intervention (an } \\
\text { exit survey) }\end{array}$ & $\begin{array}{l}\text { EORTC QLQ-C30 } \\
\text { EORTC QLQ-BR23 } \\
\text { EORTC QLQ-CR38 } \\
\text { EORTC QLQ-LC13 }\end{array}$ & $\begin{array}{l}\text { Four } \\
\text { consecutive } \\
\text { visits after } \\
\text { baseline for } \\
\text { both pre } \\
\text { (control arm) } \\
\text { and post } \\
\text { (intervention } \\
\text { arm) with a } \\
\text { two-month } \\
\text { 'wash-out' } \\
\text { period }\end{array}$ & $\begin{array}{l}\text { Patients and } \\
\text { nurses before } \\
\text { consultations }\end{array}$ & No & Yes & $* * *$ & $* * *$ & $\sqrt{ } \sqrt{ }$ \\
\hline $\begin{array}{l}\text { Mark et al. } \\
\text { (2008) [49] }\end{array}$ & $\begin{array}{l}\text { USA (Thomson } \\
\text { Healthcare, } \\
\text { Washington } \\
\text { DC) }\end{array}$ & $\begin{array}{l}\text { A cross-sectional } \\
\text { survey of the of } \\
\text { both patients' } \\
\text { and health } \\
\text { professionals' } \\
\text { experience; A } \\
\text { before-after }\end{array}$ & $\begin{array}{l}100 \text { cancer } \\
\text { patients and } 92 \\
\text { health } \\
\text { professionals } \\
\text { on the } \\
\text { experience of } \\
\text { The Patient } \\
\text { Assessment, }\end{array}$ & $\begin{array}{l}\text { Questionnaire } \\
\text { survey of } 102 \\
\text { providers The } \\
\text { patients } \\
\text { satisfaction survey } \\
(\mathrm{n}=100) \text { including } \\
8 \text {-item on PCM } \\
200 \text { patient chart }\end{array}$ & $\begin{array}{l}\text { PCM An } \\
\text { education } \\
\text { component (not } \\
\text { reported in } \\
\text { this study) }\end{array}$ & $\begin{array}{l}\text { Each visit to } \\
\text { clinic }\end{array}$ & $\begin{array}{l}\text { Clinicians } \\
\text { ( } n=\text { unreported) }\end{array}$ & No & $?$ & $* *$ & *** & $\sqrt{ } \sqrt{ }$ \\
\hline
\end{tabular}


Table 3 The characteristics of design and study quality (Continued)

\begin{tabular}{|c|c|c|c|c|c|c|c|c|c|c|c|c|}
\hline & & $\begin{array}{l}\text { patient chart } \\
\text { review }\end{array}$ & $\begin{array}{l}\text { Care and } \\
\text { Education (PACE) } \\
\text { System, including } \\
\text { PCM instrument } \\
\text { and an education } \\
\text { component }\end{array}$ & $\begin{array}{l}\text { reviews (100 } \\
\text { charts before and } \\
100 \text { charts after } \\
\text { the PACE system) }\end{array}$ & & & & & & & & \\
\hline $\begin{array}{l}\text { Kearney et al. } \\
\text { (2009) [50] }\end{array}$ & $\begin{array}{l}\text { UK (Cancer } \\
\text { Care Research } \\
\text { Centre, } \\
\text { University of } \\
\text { Stirling, Stirling) }\end{array}$ & $\begin{array}{l}\text { RCT: Control } \\
\text { group versus } \\
\text { intervention } \\
\text { group (mobile } \\
\text { phone-based } \\
\text { remote } \\
\text { monitoring of } \\
\text { symptoms) over } \\
\text { five time points }\end{array}$ & $\begin{array}{l}56 \text { patients with } \\
\text { lung, breast or } \\
\text { colorectal cancer } \\
\text { for each group } \\
\text { (total } 112 \\
\text { patients) }\end{array}$ & $\begin{array}{l}\text { Paper version of } \\
\text { electronic, Mobile } \\
\text { phone-based } \\
\text { Advanced } \\
\text { Symptom } \\
\text { Management } \\
\text { Systems (ASyMS }{ }^{\circ} \text { ) } \\
\text { based on } \\
\text { Common Toxicity } \\
\text { Criteria Adverse } \\
\text { Events (CTCAE) } \\
\text { grading system } \\
\text { and the } \\
\text { Chemotherapy } \\
\text { Symptom } \\
\text { Assessment Scale }\end{array}$ & $\begin{array}{l}\text { Mobile phone- } \\
\text { based (ASyMS }{ }^{9} \text { ) } \\
\text { including } \\
\text { chemotherapy- } \\
\text { related morbidity } \\
\text { of six common } \\
\text { symptoms } \\
\text { (nausea, vomiting, } \\
\text { fatigue, mucositis, } \\
\text { hand-foot } \\
\text { syndrome and } \\
\text { diarrhoea) }\end{array}$ & $\begin{array}{l}\text { Five times } \\
\text { including } \\
\text { baseline and } \\
\text { each of four } \\
\text { chemotherapy } \\
\text { cycles over a } \\
\text { period of } 14 \\
\text { days }\end{array}$ & $\begin{array}{l}\text { Doctors only } \\
\text { (n=unreported) }\end{array}$ & Yes & Yes & $* *$ & $* * * *$ & $\sqrt{ } \sqrt{ }$ \\
\hline $\begin{array}{l}\text { Carlson et al. } \\
\text { (2010) [51] }\end{array}$ & $\begin{array}{l}\text { Canada (Tom } \\
\text { Baker Cancer } \\
\text { Centre, } \\
\text { University of } \\
\text { Calgary, } \\
\text { Alberta) }\end{array}$ & $\begin{array}{l}\text { RCT: minimum } \\
\text { screening } \\
\text { (distress) / full } \\
\text { screening / } \\
\text { Triage : full } \\
\text { screening + } \\
\text { referring to } \\
\text { appropriate } \\
\text { services }\end{array}$ & $\begin{array}{l}585 \text { breast } \\
\text { cancer patients } \\
+549 \text { lung } \\
\text { cancer patients }\end{array}$ & $\begin{array}{l}\text { Patient distress at } \\
\text { 3-month follow- } \\
\text { up; Depression } \\
\text { and anxiety at 3- } \\
\text { month follow-up }\end{array}$ & $\begin{array}{l}\text { Minimum } \\
\text { screening: Distress } \\
\text { thermometer (DT) } \\
\text { Full screening: DT } \\
+ \text { Psychological } \\
\text { scan for cancer } \\
\text { part C (PSSCAN) }\end{array}$ & Baseline & $\begin{array}{l}\text { Screening team } \\
\text { member } \\
\text { ( } n=\text { unreported) }\end{array}$ & Yes & Yes & $* * * *$ & $* * * *$ & $\sqrt{ } \sqrt{ }$ \\
\hline $\begin{array}{l}\text { Dinkel et al. } \\
\text { (2010) [52] }\end{array}$ & $\begin{array}{l}\text { German } \\
\text { (Department of } \\
\text { Psychotherapy } \\
\text { and } \\
\text { Psychosomatic } \\
\text { Medicine, } \\
\text { Technische } \\
\text { University } \\
\text { Munchen) }\end{array}$ & $\begin{array}{l}\text { Paired } \\
\text { comparison : a } \\
\text { computerised } \\
\text { and a paper } \\
\text { version of Stress } \\
\text { Index Radio } \\
\text { Oncology (SIRO) } \\
\text { tool Prospective } \\
\text { survey }\end{array}$ & $\begin{array}{l}177 \text { cancer } \\
\text { patients in } \\
\text { study } 1,273 \\
\text { cancer patients } \\
\text { in study } 2 \\
\text { ( } \mathrm{n}=142 \text { for } \\
\text { computerised } \\
\text { version and } \\
\mathrm{n}=131 \text { for } \\
\text { paper version } \\
\text { of SIRO) } 27 \\
\text { Patients, urses/ } \\
\text { radiographs } \\
\text { and } 15 \text { physicians } \\
\text { evaluated the } \\
\text { screening } \\
\text { procedure }\end{array}$ & $\begin{array}{l}\text { Agreement } \\
\text { between } \\
\text { computer and } \\
\text { paper version of } \\
\text { SIRO Patient } \\
\text { satisfaction; Time } \\
\text { need for both } \\
\text { modes; Perceived } \\
\text { utility; Perceived } \\
\text { impact on } \\
\text { communication; } \\
\text { Perceived impact } \\
\text { on patient } \\
\text { outcome }\end{array}$ & SIRO & Any visit & $\begin{array}{l}\text { Doctors and } \\
\text { nurses } \\
\text { (n=unreported) }\end{array}$ & No & No & $* * *$ & $* *$ & $\sqrt{ } \sqrt{ }$ \\
\hline
\end{tabular}


Table 3 The characteristics of design and study quality (Continued)

\begin{tabular}{|c|c|c|c|c|c|c|c|c|c|c|c|c|}
\hline $\begin{array}{l}\text { Halkett et al. } \\
\text { (2010) [11] }\end{array}$ & $\begin{array}{l}\text { Australian } \\
\text { (WA Centre for } \\
\text { Cancer and } \\
\text { Palliative Care, } \\
\text { Curtin } \\
\text { University) }\end{array}$ & $\begin{array}{l}\text { Pilot study of } \\
\text { using computer } \\
\text { touch-screen } \\
\text { technology } \\
\text { to asses } \\
\text { psychological } \\
\text { distress in } \\
\text { patients }\end{array}$ & $\begin{array}{l}60 \text { patients } \\
\text { with various } \\
\text { gynaecological } \\
\text { cancers }\end{array}$ & $\begin{array}{l}\text { Patient } \\
\text { satisfaction with } \\
\text { both touch- } \\
\text { screen and paper } \\
\text { questionnaire; } \\
\text { Perceived utility } \\
\text { of both modes by } \\
\text { patients and } \\
\text { health } \\
\text { professionals }\end{array}$ & $\begin{array}{l}\text { EORTC QLQ-C30 } \\
\text { HADS The } \\
\text { Supportive Care } \\
\text { Needs Scale The } \\
\text { Distress } \\
\text { Thermometer } \\
\text { Follow } \\
\text { questionnaire } \\
\text { survey on } \\
\text { perceived utility } \\
\text { of both modes }\end{array}$ & Once & $\begin{array}{l}\text { Nurses and } \\
\text { doctors }\end{array}$ & Yes & Yes & * & $* *$ & $\sqrt{ }$ \\
\hline $\begin{array}{l}\text { Ruland et al. } \\
\text { (2011) [53] }\end{array}$ & $\begin{array}{l}\text { Norway (Centre } \\
\text { for Shared } \\
\text { Decision } \\
\text { Making and } \\
\text { Nursing } \\
\text { Research, } \\
\text { Oslo University } \\
\text { Hospital, Oslo) }\end{array}$ & $\begin{array}{l}\text { RCT: a } \\
\text { computer- } \\
\text { assisted, } \\
\text { interactive } \\
\text { tailored } \\
\text { assessment } \\
\text { (ITPA) with } \\
\text { feedback vs ITPA } \\
\text { only in oncology } \\
\text { practice }\end{array}$ & $\begin{array}{l}145 \text { patients } \\
\text { treated for } \\
\text { leukaemia or } \\
\text { lymphoma }\end{array}$ & $\begin{array}{l}\text { Number of } \\
\text { patient symptoms } \\
\text { and problems } \\
\text { addressed } \\
\text { Changes in } \\
\text { symptom distress } \\
\text { Changes in } \\
\text { patients' need for } \\
\text { symptom } \\
\text { management } \\
\text { support over } \\
\text { time, SF-36, } \\
\text { Center for } \\
\text { Epidemiological } \\
\text { Studies } \\
\text { Depression Scale } \\
\text { (CES-D), Medical } \\
\text { Outcome Study } \\
\text { Social Support } \\
\text { Scale (MOS - SS) }\end{array}$ & $\begin{array}{l}\text { Choice ITPA(19 } \\
\text { symptoms } \\
\text { (0-4 scale on } \\
\text { bothersome) } \\
\text { and a severity } \\
\text { scale } \\
\text { of } 0-10)\end{array}$ & $\begin{array}{l}\text { Every inpatient } \\
\text { admission with } \\
\text { up to four } \\
\text { follow-up visits }\end{array}$ & $\begin{array}{l}\text { Doctors } \\
\text { and nurses } \\
\text { ( } \mathrm{n}=\text { unreported) }\end{array}$ & $\begin{array}{l}\text { No (as see } \\
\text { appropriate) }\end{array}$ & Yes & $* * *$ & $* * * *$ & $\sqrt{ } \sqrt{ }$ \\
\hline $\begin{array}{l}\text { Velikova } \\
\text { et al. (2010) } \\
\text { [54] }\end{array}$ & $\begin{array}{l}\text { UK (Cancer } \\
\text { Research UK } \\
\text { Clinical Centre } \\
\text { - Leeds) }\end{array}$ & $\begin{array}{l}\text { RCT: } \\
\text { Intervention/ } \\
\text { control- } \\
\text { attention/control } \\
\text { in a ratio of 2:1:1 }\end{array}$ & $\begin{array}{l}286 \text { cancer } \\
\text { patients } \\
\text { commencing } \\
\text { treatment at } \\
\text { the Medical } \\
\text { Oncology Clinic } \\
\text { at St James } \\
\text { Hospital }\end{array}$ & $\begin{array}{l}\text { Medical Care } \\
\text { Questionnaire } \\
\text { (MCQ): } 15 \text {-item } \\
\text { three subscales: } \\
\text { Communication, } \\
\text { Coordination, } \\
\text { Patient } \\
\text { preferences } \\
\text { Satisfaction with } \\
\text { care Patients' and } \\
\text { physicians' } \\
\text { evaluation of the } \\
\text { intervention K- } \\
\text { index (Continuity } \\
\text { of care: } \\
\text { K=(number of } \\
\text { visits - number of } \\
\text { doctors)/(number } \\
\text { of visits - }-1 \text { ). }\end{array}$ & $\begin{array}{l}\text { EORTC QOQ-C30 } \\
\text { Hospital Anxiety } \\
\text { and Depression } \\
\text { Scale (HADS) }\end{array}$ & $\begin{array}{l}\text { Regular clinic } \\
\text { visit over an } \\
\text { average of } 6 \\
\text { months }\end{array}$ & Doctor $(n=28)$ & No & $\begin{array}{l}\text { One to one } \\
\text { training and } \\
\text { manual } \\
\text { provided }\end{array}$ & $* * *$ & $* * * *$ & $\sqrt{ } \sqrt{ }$ \\
\hline
\end{tabular}


Table 3 The characteristics of design and study quality (Continued)

\begin{tabular}{|c|c|c|c|c|c|c|c|c|c|c|c|c|}
\hline $\begin{array}{l}\text { Bainbridge } \\
\text { et al.(2011) [55] }\end{array}$ & $\begin{array}{l}\text { Canada } \\
\text { (Juravinski } \\
\text { Cancer Centre, } \\
\text { McMaster } \\
\text { University, } \\
\text { Hamilton, } \\
\text { Ontario) }\end{array}$ & $\begin{array}{l}\text { Survey on } \\
\text { the utility of }\end{array}$ & $\begin{array}{l}128 \text { nurses, } \\
\text { physicians, and } \\
\text { allied health } \\
\text { professionals }\end{array}$ & $\begin{array}{l}\text { Perceptions of } \\
\text { use and utility of } \\
\text { the Edmonton } \\
\text { Symptom } \\
\text { Assessment } \\
\text { System (ESAS) } \\
\text { adopted by } \\
\text { Ontario's cancer } \\
\text { centres since } \\
2007\end{array}$ & ESAS & Every clinic visit & $\begin{array}{l}\text { Doctors and } \\
\text { nurses }\end{array}$ & Yes & Yes & $*$ & $*$ & $\sqrt{ }$ \\
\hline \multirow[t]{3}{*}{$\begin{array}{l}\text { Berry et al. } \\
\text { (2011) [56] }\end{array}$} & $\begin{array}{l}\text { USA (Dana- } \\
\text { Faber Cancer } \\
\text { Institute, } \\
\text { Boston) }\end{array}$ & $\begin{array}{l}\text { RCT: } \\
\text { Intervention / } \\
\text { Control }\end{array}$ & $\begin{array}{l}660 \text { cancer } \\
\text { patients with } \\
\text { various cancer } \\
\text { diagnoses and } \\
\text { stages at two } \\
\text { institutions of a } \\
\text { comprehensive } \\
\text { cancer centre }\end{array}$ & $\begin{array}{l}\text { 1.Audio-recorded } \\
\text { content of all } \\
\text { communication } \\
\text { between } \\
\text { clinicians, } \\
\text { patients and } \\
\text { accompanying } \\
\text { friends or family } \\
\text { members at each } \\
\text { T2 visit (4- } 6 \text { wks } \\
\text { after the } \\
\text { treatment) }\end{array}$ & $\begin{array}{l}\text { Patient reported } \\
\text { symptoms and } \\
\text { quality-of-life } \\
\text { (SQLIs) from the } \\
\text { Electronic } \\
\text { Self-Report } \\
\text { Assessment- } \\
\text { Cancer (ESRA-C) }\end{array}$ & $\begin{array}{l}\text { Every clinic visit } \\
\text { during the } \\
\text { study period }\end{array}$ & $\begin{array}{l}\text { Doctors } \\
\text { ( } \mathrm{n}=76 \text { principle } \\
\text { physicians and } \\
\text { other) or } \\
\text { incorporated } \\
\text { into charts } \\
\text { ( } \mathrm{n}=\text { unreported) }\end{array}$ & No & Yes & $* * *$ & $* * * *$ & $\sqrt{ } \sqrt{ }$ \\
\hline & & & & $\begin{array}{l}\text { 2.Clinic visit } \\
\text { duration }\end{array}$ & & & & & & & & \\
\hline & & & & $\begin{array}{l}\text { 3. Physician exit } \\
\text { questionnaire } \\
\text { survey }\end{array}$ & & & & & & & & \\
\hline $\begin{array}{l}\text { Cleeland } \\
\text { et al. (2011) } \\
\text { [20] }\end{array}$ & $\begin{array}{l}\text { USA (MD } \\
\text { Anderson } \\
\text { Cancer Center, } \\
\text { The University } \\
\text { of Texas) }\end{array}$ & $\begin{array}{l}\text { RCT: e-mail alert } \\
\text { of symptom to } \\
\text { patients' clinical } \\
\text { team versus no } \\
\text { e-mail alert }\end{array}$ & $\begin{array}{l}79 \text { lung cancer } \\
\text { patients } \\
\text { receiving } \\
\text { thoracotomy }\end{array}$ & $\begin{array}{l}\text { 1. Four targeted } \\
\text { symptom: pain, } \\
\text { distress, disturbed } \\
\text { sleep, and } \\
\text { shortness of } \\
\text { breath, } \\
\text { constipation (no } \\
\text { fatigue as no } \\
\text { effective } \\
\text { response) 2. MD } \\
\text { Anderson } \\
\text { Symptom } \\
\text { Inventory (MDASI) } \\
\text { at follow-up clinic } \\
\text { visit 3. An exit } \\
\text { questionnaire } \\
\text { survey }\end{array}$ & $\begin{array}{l}\text { Automated } \\
\text { telephone calls } \\
\text { (IVR system): } \\
\text { MDASI (13 } \\
\text { common cancer } \\
\text { related } \\
\text { symptoms) }\end{array}$ & $\begin{array}{l}\text { Twice weekly, } \\
\text { up to } 4 \text { wks } \\
\text { after discharge }\end{array}$ & $\begin{array}{l}\text { Nurses } \\
\text { ( } n=\text { unreported) }\end{array}$ & Yes & $\begin{array}{l}\text { Training to } \\
\text { patients } \\
\text { provided }\end{array}$ & $* *$ & $* * * *$ & $\sqrt{ } \sqrt{ }$ \\
\hline $\begin{array}{l}\text { Takeuchi } \\
\text { et al. (2011) } \\
\text { [57] }\end{array}$ & $\begin{array}{l}\text { UK (St James's } \\
\text { Institute of } \\
\text { Oncology, } \\
\text { Leeds) }\end{array}$ & $\begin{array}{l}\text { Longitudinal } \\
\text { study of data as } \\
\text { part of Velikova } \\
\text { et al. (2004, } \\
\text { 2010) RCT }\end{array}$ & $\begin{array}{l}286 \text { cancer } \\
\text { patients } \\
\text { commencing } \\
\text { treatment at } \\
\text { the Medical }\end{array}$ & $\begin{array}{l}\text { Audio-recorded } \\
\text { content of } \\
\text { Patient-physician } \\
\text { communication: } \\
\text { Longitudinal }\end{array}$ & $\begin{array}{l}\text { EORTC QOQ-C30 } \\
\text { Hospital Anxiety } \\
\text { and Depression } \\
\text { Scale (HADS) }\end{array}$ & $\begin{array}{l}\text { Four } \\
\text { consecutive } \\
\text { visits from } \\
\text { baseline }\end{array}$ & Doctor $(n=28)$ & No & $\begin{array}{l}\text { One to one } \\
\text { training and } \\
\text { manual } \\
\text { provided }\end{array}$ & $* * *$ & $* * * *$ & $\sqrt{ } \sqrt{ }$ \\
\hline
\end{tabular}


Table 3 The characteristics of design and study quality (Continued)

$\begin{array}{ll}\text { Oncology Clinic } & \text { impact of PRO } \\ \text { at St James } & \text { intervention; } \\ \text { Hospital } & \text { dynamics of } \\ & \text { communication; } \\ & \text { association } \\ & \text { between severity } \\ & \text { of symptoms/ } \\ & \text { functions and } \\ & \text { clinic discussion }\end{array}$

*: Four stars indicate a randomised trial or experimental study; 3 stars indicate a controlled trial, pre-post trial with control (controlled before-after trial), time series, or observational cohort with multivariable

adjustment; 2 stars indicate a pre-post trial without control, observational cohort study without multivariable adjustment, cross-sectional study without multivariable adjustment, analysis of time trends without control,

or well-designed qualitative study; and 1 star indicates a case series, other qualitative study, or survey (descriptive) study.

$\checkmark$ : Three checks indicate great weight in the stratum's body of evidence, 2 checks indicate moderate weight, and 1 check indicates little weight.

\#: EORTC QLQ-C30: European Organisation for Research and Treatment of Cancer Quality of Life (EORTC QLQ) Core Questionnaire; SF-36: The Short Form(36) Health Survey; FACT-G: Functional Assessment of Cancer Therapy-general; EORTC QLQ-BR23: EORTC QLQ Breast Cancer Scale; EORTC QLQ-CR38: EORTC QLQ Colorectal Cancer Scale; EORTC QLQ-LC; EORTC QLQ Lung Cancer Scale; NCI CATAE: National Cancer Institute

Common Terminology Criteria for Adverse Events; FACT-BCS: Functional Assessment for Cancer Therapy-Breast Cancer Subscale; FACT-C: Functional Assessment of Cancer Therapy-Colorectal Quality of Life Instrument; FACT-L: Functional Assessment of Cancer Therapy-Lung Cancer Subscale; MOS-SS: Medical Outcomes Study Social Support Survey; GDS: Geriatric Depression Scale (short form); QARSQ-PH: Physical Health subscale of the Older American Resources and Services Questionnaire (OARSQ); UMPSI: Utilisation of Mental Health and Psychosocial Services Instrument; GSRE: Geriatric Schedule of Recent Experience Instrument;

?: No data or unable to classify data available. 
Table 4 The components, rating criteria and symbol, and categories used in summarising the study evidence in the current study

\begin{tabular}{|c|c|c|c|}
\hline & Domain 1 & Domain 2 & Global (GRADE) \\
\hline $\begin{array}{l}\text { Decision } \\
\text { Components }\end{array}$ & $\begin{array}{l}\text { Subject of public reporting (or } \\
\text { study population) and study } \\
\text { participants (sample) }\end{array}$ & Types of study (i.e. study designs) & $\begin{array}{l}\text { Components from Domain } 1 \& 2 \text { as well as } \\
\text { implementation and adherence to intervention, dose- } \\
\text { response gradient, precision and validity of the } \\
\text { outcomes, uncertainty of direction of the results. }\end{array}$ \\
\hline Rating criteria & $\begin{array}{l}\text { How well does the study sample } \\
\text { represent the study population? }\end{array}$ & $\begin{array}{l}\text { How strong is the study design } \\
\text { both in terms of its external and } \\
\text { internal validity? }\end{array}$ & $\begin{array}{l}\text { How much weight does the current study add to the } \\
\text { evidence-base taking into considerations of all the } \\
\text { components above? }\end{array}$ \\
\hline \multirow{4}{*}{$\begin{array}{l}\text { Symbol used \& } \\
\text { categories of } \\
\text { rating }\end{array}$} & $1 *$ : no overlap & $1^{*}$ : weakest design & $\sqrt{ }$ : little weight \\
\hline & $2^{*}$ : modest overlap & $2^{*}:$ moderate design & $\sqrt{ } \sqrt{ }:$ moderate weight \\
\hline & $3^{*}$ : large overlap & $3^{*}$ : strong design & $\sqrt{ } \sqrt{ }$ : great weight \\
\hline & 4*: complete overlap & $4^{*}$ : strongest design & \\
\hline
\end{tabular}

reviews, it was listed in the summation tables for the purpose of comparison.

\section{Overview of study quality}

There has been a marked increase in the volume and quality of the studies published recently in this area. Of the 16 randomised controlled trials included in this review, 7 were published between 2010 and 2011. The quality of studies published since 2010 is also demonstrably improved with much larger sample sizes, including 3 trials $[54,57,61]$ with a sample size greater than 200 and 2 trials with a sample size over $580[51,56]$.

However, despite the increased volume and improved quality of the studies, there remains a lack of large cluster randomised controlled design studies, as recommended by Fayers [62] who argues that cluster RCTs are well suited to overcome the limitations of simple RCTs. It is well-known that system intervention trials such as routine collection of PROs, and feedback to the clinicians and systems, are prone to crosscontamination and to introducing investigator and participant biases. Two recently published studies [54,57] were the continuation of an earlier study published by Velikova et al. (2004) [36]. Most studies reviewed did not systematically examine outcomes and mechanisms, and placed more emphasis on processes rather than outcome measures [25]. All studies were conducted in a limited setting (often in a single centre) thus restricting the generalisation of the findings.

No studies have adopted a comprehensive theoretical model and framework, despite the repeated demand from leading researchers in the area [25,63-65]. All studies focused on the patient and health professional level within the clinic setting. No study to date has examined the impact of collecting PROs on health care organisations, health system improvement, quality improvement or population health at a system or societal level.

\section{Overview of study findings}

\section{Impact on patient-provider communication}

Across the 27 studies included in this review, 4 studies $[39,47,51,53]$ did not examine or report the effect of a routinely collected PRO on patient-provider communication. Among the 23 studies that did report such an impact, 21 studies (91.3\%) reported a positive effect which included well-designed and conducted large RCTs [33,36,37,54,56,57]. One study reported no significant improvement of patientprovider communication possibly due to a lower severity level of cancer patients (only 37\% of patients received anticancer therapy, hence the reduced need for communication for the treatment) [38]. Another study reporting a negative effect had an already high communication level at baseline (hence a ceiling effect leaving little room for further improvement) [34].

\section{Impact on monitoring treatment response}

Despite most of included 27 studies did not explicitly state their study objectives as to examine the impact on monitoring treatment response, 11 of the 27 studies did report an outcome (Table 3) [16,20,36,41,45-51]. All 11 studies found a strong or modest effect of implementing PROs on the increased monitoring activities of treatment response. The strongest effect occurred in the studies that focused on the monitoring of patient symptoms, side effects and toxicity during and after chemotherapy for the outpatients. In particular, the real-time, patient reported symptoms and toxicity (through innovative mobile phone-based, web-based or IVR systems) significantly improved the monitoring of treatment response.

\section{Impact on detecting unrecognised problems}

Although the idea of routinely collected PROs may provide better opportunities for services providers (as well as patients) to detect unrecognised problems through growing awareness, improved communication and monitoring seems 
Table 5 The impact and effect sizes of the studies on patients, care providers and organisations*

\begin{tabular}{|c|c|c|c|c|c|c|c|c|c|}
\hline Studies & $\begin{array}{l}\text { Doctor-patient } \\
\text { communication }\end{array}$ & $\begin{array}{l}\text { Monitor } \\
\text { treatment } \\
\text { response }\end{array}$ & $\begin{array}{l}\text { Detect } \\
\text { unrecognised } \\
\text { problems }\end{array}$ & $\begin{array}{l}\text { Changes } \\
\text { to patient health behaviour }\end{array}$ & $\begin{array}{l}\text { Changes to } \\
\text { patient } \\
\text { management }\end{array}$ & $\begin{array}{l}\text { Improved } \\
\text { patient } \\
\text { satisfaction }\end{array}$ & $\begin{array}{l}\text { Improved } \\
\text { health outcomes }\end{array}$ & $\begin{array}{l}\text { Feasibility } \\
\text { of the } \\
\text { implementation }\end{array}$ & $\begin{array}{l}\text { Moderating and } \\
\text { subgroup effect }\end{array}$ \\
\hline $\begin{array}{l}\text { Trowbridge } \\
\text { et al. (1997)[39] }\end{array}$ & & & ++ & & ++ & & $\begin{array}{l}+ \text { (but no change } \\
\text { in PMI) }\end{array}$ & +++ & \\
\hline $\begin{array}{l}\text { Tazenzer et al. } \\
\text { (2000)[33] }\end{array}$ & +++ & & ++ & & + & - & & +++ & \\
\hline $\begin{array}{l}\text { McLachlan } \\
\text { et al. (2001)[38] }\end{array}$ & $\begin{array}{l}\text {-(no time } \\
\text { differences in } \\
\text { consultation } \\
\text { between two } \\
\text { arms) }\end{array}$ & & $\begin{array}{l}\text {-(only } 37 \% \\
\text { patients } \\
\text { receiving } \\
\text { anticancer } \\
\text { therapy at } \\
\text { baseline) }\end{array}$ & & & - & - & +++ & $\begin{array}{l}+ \text { (on high BDI score } \\
\text { subgroup) }\end{array}$ \\
\hline \multirow[t]{2}{*}{$\begin{array}{l}\text { Detmar et al. } \\
\text { (2002)[37] }\end{array}$} & \multirow{2}{*}{$\begin{array}{l}+++(10 \text { out of } \\
12 \text { HRoL } \\
\text { measures, } \\
\text { especially on } \\
\text { social } \\
\text { functioning and } \\
\text { fatigue) }\end{array}$} & & \multirow[t]{2}{*}{++} & & \multirow{2}{*}{$\begin{array}{l}+ \text { (increased } \\
\text { patient } \\
\text { counselling })+( \\
25 \% \text { with family } \\
\text { members and } \\
\text { primary care } \\
\text { physicians) }\end{array}$} & $\begin{array}{l}+ \text { (emotional } \\
\text { support) }\end{array}$ & \multirow[t]{2}{*}{$+(\mathrm{SF}-36)$} & \multirow[t]{2}{*}{+++} & \multirow{2}{*}{$\begin{array}{l}\text { + (before-after } \\
\text { improvement by } \\
\text { intervention group) }\end{array}$} \\
\hline & & & & & & $\begin{array}{l}++ \text { (information } \\
\text { sharing \& } \\
\text { communication) }\end{array}$ & & & \\
\hline $\begin{array}{l}\text { Mooney et al. } \\
\text { (2002)[16] }\end{array}$ & +++ & ++ & ++ & & & +++ & & ++ & \\
\hline $\begin{array}{l}\text { Velikova et al. } \\
\text { (2004)[36] }\end{array}$ & +++ & & $\begin{array}{l}++(64 \% \\
\text { encounters } \\
\text { involving } \\
\text { referring to } \\
\text { HRoL by } \\
\text { physicians) }\end{array}$ & & $\begin{array}{l}\text {-(possible due to } \\
\text { simple coding } \\
\text { between two arms) } \\
\text { +(contributed to } \\
\text { patient } \\
\text { management in } \\
11 \% \text { of encounters } \\
\text { intervention arm). }\end{array}$ & & $\begin{array}{l}++ \text { (overall quality } \\
\text { of life and } \\
\text { emotional } \\
\text { functioning) }\end{array}$ & $\begin{array}{l}++(\text { response } \\
\text { rate } 70 \%)\end{array}$ & $\begin{array}{l}+ \text { (more discussion of } \\
\text { HRoL subgroup had } \\
\text { better outcome within } \\
\text { intervention group) }\end{array}$ \\
\hline $\begin{array}{l}\text { Basch et al. } \\
\text { (2005)[41] }\end{array}$ & +++ & + & ++ & & & +++ & + & $\begin{array}{l}++(65 \% \text { patient } \\
\text { log in before } \\
\text { any verbal } \\
\text { encouragement) }\end{array}$ & \\
\hline $\begin{array}{l}\text { Boyes et al. } \\
\text { (2006)[35] }\end{array}$ & $\begin{array}{l}+(50 \% \\
\text { oncologists in } \\
\text { intervention } \\
\text { group talked } \\
\text { with patients) }\end{array}$ & & & & - & & $\begin{array}{l}++ \text { (fewer } \\
\text { deliberating } \\
\text { symptoms) - } \\
\text { (anxiety and } \\
\text { depression) }\end{array}$ & + & - \\
\hline $\begin{array}{l}\text { Hoekstra et al. } \\
\text { (2006)[42] }\end{array}$ & $\begin{array}{l}+/-(\text { Only } 18 \% \\
\text { patients used it } \\
\text { enhancing } \\
\text { communication) }\end{array}$ & & & - & & & $\begin{array}{l}++ \text { (lower } \\
\text { prevalence in } 9 \\
\text { out of } 10 \\
\text { symptoms; } \\
\text { deteriorated less } \\
\text { in } 8 \text { out of } 10 \\
\text { symptoms) }\end{array}$ & + & $\begin{array}{l}\text { The beneficial effects } \\
\text { were pronounced in the } \\
\text { deteriorated group. }\end{array}$ \\
\hline
\end{tabular}


Table 5 The impact and effect sizes of the studies on patients, care providers and organisations* (Continued)

Korniblith et al. +++ (both arms)

(2006)[43]

$++($ more

from TM+EM

arm)

Basch et al.

(2007)[44]

Rosenbloom -(Possible

et al. (2007)[34] Ceiling effect)

Weaver et al. + (nurse-patient + (2007)[45] communication)

Butt et al.

(2008)[46]

Given et al.

(2008)[47]

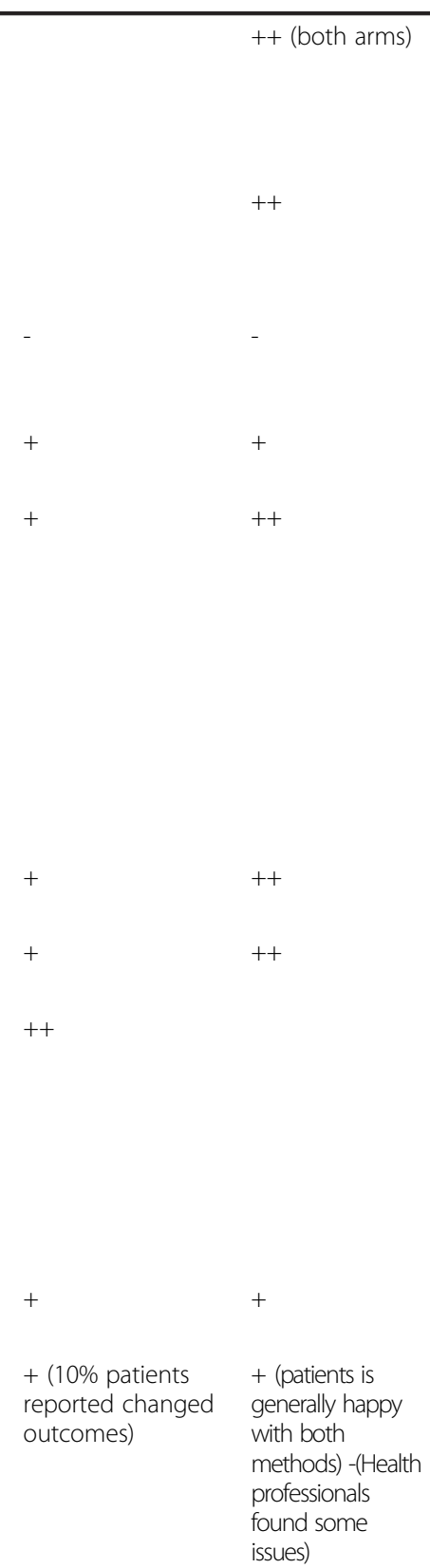

++ (better

+ EM arm -

reduction of

psychological

distress)

$++$

++ (can be

improved

through

reminder)

$\begin{array}{ll}-\quad & \text { No effect even among } \\ \text { the most highly } \\ \text { distressed patients }\end{array}$

$++$

$++$

++ (ATSM more

likely to

responses in

symptom

management and

required less time

to do so)

distressed patients

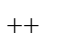

combination

chemotherapy protocols,

those patients treated

with single agent had

greater response and

shorter time to

response)
Hilarius et
(2008)[48]

Mark et al

(2008)[49]

Kearney et al. +

(2009)[50]

Carlson et al.

(2010)[51]

Dinkel et al.

(2010)[52]

Halkett et al. + (around 25\%

(2010)[11] of doctors) 
Table 5 The impact and effect sizes of the studies on patients, care providers and organisations* (Continued)

Ruland et al. $++\quad++++$

(2010)[53]

$++$

$++$

(no difference in $\quad++(86 \%$ in

coordination of intervention vs

care \& 'preferences $29 \%$ in the

to see usual attention-

doctor' subscale) control group)

et al. (2011) [55]

Berry et al $\quad++(25 \%$

(2011)[56] physician

physician
explicitly

referred to SQL

summary)

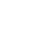

Cleeland et al. ++

$+$

Takeuchi et al. ++ (on

(2011)[57] symptom but

$$
\begin{aligned}
& \text { symptom but } \\
& \text { not function) }
\end{aligned}
$$

Note: +++ very strong effect; ++ strong effect; + some effect; +/- uncertain effect; - No effect; blank : untested or reported;

Note: +++ very strong effect; ++ strong effect; + some effect; +/- uncertain effect; - No effect; blank : untested or reported; *: Impacts on quality improvement, increased transparency, accountability, public reporting, better population and system performance (monitoring, planning, financing, evaluating, etc) were not listed due to lack of data.
$+89 \%$ of nurses and

$55 \%$ of physicians

referred to the ESAS in

clinics 'always' or ' most

of the time'

++ (the treatment effect

on communication is

evident on over

threshold group on

cognitive function,

impact on sex and social

function) 


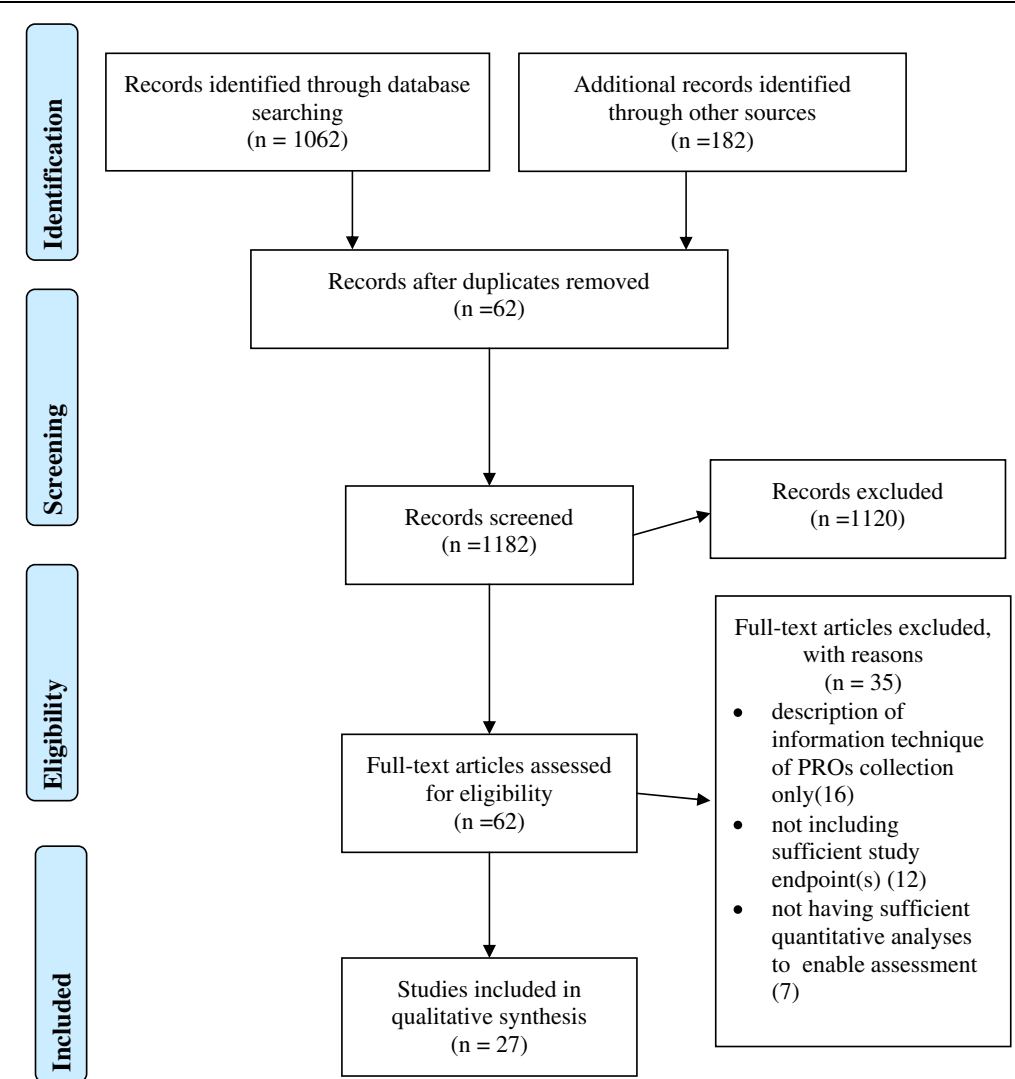

Figure 3 PRISMA Flow Diagram illustrating the systematic review process from electronic searching through to study inclusion.

intuitively plausible, only 16 out of 27 studies reported some results related to the detection of unrecognised problems (Table 3). Amongst the 16 studies, 15 studies [16,20,33,36, $37,39,41,43,45-50,53]$ reported either a strong or moderate positive impact on detecting unrecognised problems. However, a study by McLachlan and colleagues [38] did not find any difference between the intervention arm and control arm.

\section{Impact on changes to patient health behaviour}

No study to date has provided a systematic evaluation on the impact of collecting PROs on changes to patient health behaviour. It is unknown whether and how patient health behaviours have been changed.

\section{Impact on changes to patient management}

Amongst 17 studies that provided some results of changes to patient management, 13 studies [11,20,33,37,39, $45,46,48-50,52,53,55]$ reported either a strong or modest positive effect on the changes to patient management whilst 4 studies [34-36,54] found no such effect. However, it is worth noting that 10 studies did not provide any information about the changes to patient management and there were often less complete descriptions of the results on patient management when reported.

\section{Impact on patient satisfaction}

Among the 16 studies that reported results related to the impact on patient satisfaction, 13 studies $[11,16,20$, $37,41,43-46,48,49,52,54]$ reported a very strong to moderate positive effect on improved patient satisfaction. For the three studies $[33,34,38]$ that did not find such a positive effect, one study [33] reported a possible ceiling effect meaning that both the intervention group and control group had a very high baseline patient satisfaction level potentially impeding any demonstration of a significant difference between two arms during the follow-up period.

\section{Impact on health outcomes}

Amongst the 15 studies that reported some results related to the impact on health outcomes, 13 studies [20,35-37,39,41-43,45,47,50,51,53] reported some positive improvement, ranging from modest to strong, while two studies [34,38] failed to find any such effect. It appears that symptoms, side effects and toxicity are most 
likely to be improved, followed by emotional wellbeing. There is little evidence on the improvement of both overall HRQOLs as well as social wellbeing.

Impact on quality improvement, transparency, accountability and public reporting, and on better system performances (monitoring, planning, financing, evaluating, responding)

No study to date has provided a meaningful, explicit framework nor relevant evidence on these endpoints.

\section{Overall strength and direction of evidence}

Overall, there is strong evidence supporting the notion that routinely collected PROs, with feedback, improves patient-provider communication and increases patient satisfaction (Table 6). There is some evidence to support the notion that it improves the monitoring of treatment responses and detection of unrecognised problems, and there is weak but positive evidence that, over time, it leads to changes in patient management. Despite some encouraging results, there is still a great degree of uncertainty regarding the impact of routinely collected PROs, with feedback, on patient health outcomes. There is little or no evidence that it has led to significant positive improvements in quality improvement, transparency, accountability, and public reporting, or in system performance at a population health or societal level. Apart from clinical trials and clinical practice, its impact on health services research and population health is largely unknown.

\section{Potential moderating factors and links between routine PRO collection (with feedback) and patient outcomes}

Although the evidence is limited, it appears that routine collected PROs with sufficient intensity of feedback (multiple times over a sustained period of time)

Table 6 The overall strength and direction of evidence

\begin{tabular}{ll}
\hline Results & $\begin{array}{l}\text { Strength and } \\
\text { direction of evidence }\end{array}$ \\
\hline Patient-provider communication & +++ \\
Monitor treatment response & ++ \\
Detect unrecognised problems & ++ \\
Changes to patient health behaviour & $\mathrm{n} / \mathrm{a}$ \\
Changes to patient management & + \\
Improved patient satisfaction & +++ \\
Improved health outcomes & $+/ 0$ \\
Strong \& effective quality improvement & $\mathrm{n} / \mathrm{a}$ \\
Increased transparency, accountability, public & $\mathrm{n} / \mathrm{a}$ \\
reporting & \\
Better system performance (monitoring, \\
planning, financing, evaluating, responding)
\end{tabular}

Note: ++++: the strongest positive effect; $x x x x$ : the strongest negative effect; n/a: not available; 0 : mutual (no significant effect).
$[13,39,44,54]$, targeting multiple stakeholders (doctors, nurses, allied health workers, as well as patients) [35,52] with simple, clear, graphical and longitudinal meaningful interpretation of the results, and providing sufficient training for both health professionals and patients $[20,57]$, are critical links between an intervention and the intended outcomes. There is also evidence to suggest that for some complex issues such as depression and low social functioning, routine screening and feedback may need to be integrated with other strategies such as decision-making aids, education, clear management plans and clinical pathways including referrals, in order to change patient outcomes $[43,49,51]$. There is preliminary evidence that some of the impacts of PROs may be more pronounced amongst subgroups with more severe problems at baseline (e.g. depression, symptoms) $[38,42,65]$. More studies are needed to fully understanding these mediating and moderating effects.

\section{Discussion}

There is very strong evidence in supporting the notion that routine collected PROs with timely feedback enhances patient-provider communication. This current study finding of a positive effect on patient-provider communication is consistent with previous reviews conducted in both cancer [26] and non-cancer settings $[25,27,28]$. There is also strong evidence to support the notation that routine collected PROs significantly improved the monitoring of treatment response.

There is reasonably strong evidence in supporting the notation that routine collected PROs are helpful in identifying unrecognised problems in a large variety of settings. Within studies that reported, to some extent, the results related to unrecognised problems, there is a need for the development of more comprehensive and valid measures. Such a change in the measures would contribute towards understanding specifically the PROs' impact on identifying the underreported and unrecognised problems for different cancer patients at different settings.

Overall, there is reasonable evidence in favouring the hypothesis that implementing a routine collected PROs system brings positive changes to patient management in the settings where a patient management plan is integrated with a routine collection of PROs. It appears that the simple routine feedback of PROs may not be sufficient to improve patient management and outcomes [48]. Other necessary resources may be needed such as education, referral services and a detailed patient management plan following the PROs [43]. There is also a need to develop better measures of change to patient management as it is often complex and difficult to quantify [57].

There is strong evidence to support the notation that routine collected PROs with timely feedback significantly enhance patient's experience and satisfaction. There may 
be other improved experience and satisfaction in other stakeholders such as patients' family members, caregivers, as well as health professionals that were not measured or unreported. Future research into furthering the understanding of stakeholder experience after implementing routine collected PROs would be desirable.

Although positive evidence in supporting the notion that routine collected PROs may improve health outcomes is weak, this finding needs to be confirmed by better designed studies covering a large set of welldeveloped outcome measures. There is also a need to understand the impact on long-term health outcomes such as survival rate. Most of the studies included in this review did not focus on health outcomes and some of the positive improvement on the outcomes only occurred on selective measures. It is not clear how these positive improvements can be generalised across different settings.

There is a variety of models on how to routinely collect PROs and how to feed back the data to different stakeholders. Given that cancer patients are vastly different given their background, type and stage of cancer, prognosis, treatment, and the positions at the life course continuum, precaution should be exercised when attempting to apply the general observation above to each and every different setting. For example, recent studies demonstrated a positive impact of routine collected PROs on symptom control through either webbased or mobile phone based approach. However, such positive impacts were less pronounced on HRQOL.

\section{Limitations of the current review}

Our review has several limitations. First, there was no attempt made to contact the authors to ask for potential unpublished data on the topic. Thus, there may be chance of missing some grey literature or the studies that under preparation for publication. Second, given the multitude of endpoints included, and different types of studies involved, the assessment of eligibility for inclusion of potential studies required some degree of subjective judgement. Third, our application of GRADE system was rather simplistic restricted by large number of endpoints and variability of studies included. These limitations may give rise to some uncertainty in terms of synthesis of the results. Fourth, our study follows a systematic review approach with inclusion of both experimental trials and quantitative observational studies. However, we did not include qualitative studies in our review which may provide additional insight into the questions raised. This is particularly relevant with respect to questions 2 and 3 as there was very little quantitative evidence from the included studies. It is important to note that despite efforts to formulate the review endpoints based on solid and well-established causal and theoretical frameworks (providing insight into not only if but also how the introduction of PROs affects patient outcomes), the causal mechanisms and process endpoints included in the current review are by no means exhaustive. There may be other important causal mechanisms that could be benefited from a realist review approach [66].

\section{Conclusions}

There is growing evidence supporting the routine collection of PRO to enable better and patient-centred care, especially in cancer settings. Despite the strong evidence in supporting the notion that the well-implemented routine collection of PROs enhances patient-provider communication and improves patient satisfaction, and growing evidence supporting ideas that it also improves the monitoring of treatment response and the detection of the unrecognised problems, the evidence-base was weak for its impact on changes to patient management and improved health outcomes and non-existent for changes to patient health behaviour, strong and effective quality improvement, increased transparency, accountability, public reporting and better health care system performance. These evidence gaps require further committed and well-planned research in addition to the well-accepted PROs. Decision-making agencies have been well positioned for leverage on the rapid advancement of different PRO models, the application of the item response theory and computer adapted test in developing PROs, and on the acceptance of such technology by patients and health professionals over the last decade. The real-time and routinely collected PROs will enable the development of a rapid learning health system with the potential to advance our knowledge of drug development, unsurpassed models of cancer patient care and a more patient-centred health care system.

\section{Additional file}

Additional file 1: Appendix 1. Full text search strategies used in Scopus.

\section{Competing interests}

The authors declare that they have no competing interests.

\section{Authors' contributions}

JC contributed to the conceptualisation and design of the study. JC, LO, SH conducted the review. JC prepared the first draft of the paper and all other authors contributed to and approved the final manuscript.

\section{Acknowledgements}

This article is based on An Evidence Check rapid review brokered by the Sax Institute (http://www.saxinstitute.org.au) for the Cancer Institute NSW, 2012. The views expressed in this article are entirely those of the authors and should not be interpreted as that of the Sax Institute or the Cancer Institute NSW. 
Received: 31 October 2012 Accepted: 28 May 2013

Published: 11 June 2013

\section{References}

1. Jones JB, Snyder CF, Wu AW: Issues in the design of Internet-based systems for collecting patient-reported outcomes. Qual Life Res 2007, 16(8):1407-1417.

2. Guidance for Industry Patient-Reported Outcome Measures: Use in Medical Product Development to Support Labeling Claims. Silver Spring, Maryland: U.S. Department of Health and Human Services Food and Drug Administration; 2009.

3. Ousey K, Cook L: Understanding patient reported outcome measures (PROMs). Br J Community Nurs 2011, 16(2):80-82.

4. Henly SJ: The promise of PROMIS. Nurs Res 2010, 59(2):77.

5. Fromme EK, Kenworthy-Heinige T, Hribar M: Developing an easy-to-use tablet computer application for assessing patient-reported outcomes in patients with cancer. Support Care Cancer 2011, 19(6):815-822.

6. Fiscella K, Ransom S, Jean-Pierre P, Cella D, Stein K, Bauer JE, Crane-Okada R, Gentry S, Canosa R, Smith T, et al: Patient-reported outcome measures suitable to assessment of patient navigation. Cancer 2011, 117(SUPPL. 15):3603-3617.

7. Burgess DJ, Powell AA, Griffin JM, Partin MR: Race and the validity of selfreported cancer screening behaviors: Development of a conceptual model. Prev Med 2009, 48(2):99-107.

8. Jayadevappa R, Johnson JC, Chhatre S, Wein AJ, Malkowicz SB: Ethnic variation in return to baseline values of patient-reported outcomes in older prostate cancer patients. Cancer 2007, 109(11):2229-2238.

9. Sloan JA, Frost MH, Berzon R, Dueck A, Guyatt G, Moinpour C, Sprangers M, Ferrans C, Cella D, Aaronson N, et al: The clinical significance of quality of life assessments in oncology: A summary for clinicians. Support Care Cancer 2006, 14(10):988-998.

10. Henly SJ: Editorial: The promise of PROMIS. Nurs Res 2010, 59(2):77.

11. Halkett $G$, Jiwa $M$, Tanner $P$, Fournier $C$, Katris $P$ : Trialling computer touch-screen technology to assess psychological distress in patients with gynaecological cancer. Australasian Medical Journal 2010, 3(12):781-785.

12. Clark K, Bardwell WA, Arsenault T, DeTeresa R, Loscalzo M: Implementing touch-screen technology to enhance recognition of distress. Psychooncology 2009, 18(8):822-830.

13. Velikova G: Use of electronic quality of life applications in cancer research and clinical practice. Expert Rev Pharmacoecon Outcomes Res 2004, 4(4):403-411.

14. Kilbourn KM, Bargai N, Durning PE, Deroche K, Madore S, Zabora J: Validity of the psycho-oncology screening tool (POST). J Psychosoc Oncol 2011, 29(5):475-498

15. Macvean ML, White VM, Pratt S, Grogan S, Sanson-Fisher R: Reducing the unmet needs of patients with colorectal cancer: A feasibility study of the Pathfinder Volunteer Program. Support Care Cancer 2007, 15(3):293-299.

16. Mooney KH, Beck SL, Friedman RH, Farzanfar R: Telephone-linked care for cancer symptom monitoring: A pilot study. Cancer Pract 2002, 10(3):147-154.

17. Forbat L, Maguire R, McCann L, llingworth N, Kearney N: The use of technology in cancer care: Applying Foucault's ideas to explore the changing dynamics of power in health care. J Adv Nurs 2009, 65(2):306-315.

18. Matthew AG, Currie KL, Irvine J, Ritvo P, Santa Mina D, Jamnicky L, Nam R, Trachtenberg J: Serial personal digital assistant data capture of healthrelated quality of life: A randomized controlled trial in a prostate cancer clinic. Health Qual Life Outcomes 2007, 5:38. http://dx.doi.org/10.1186/14777525-5-38.

19. Williams PD, Williams K, Lafaver-Roling $S$, Johnson R, Williams AR: An intervention to manage patient-reported symptoms during cancer treatment. Clin J Oncol Nurs 2011, 15(3):253-258.

20. Cleeland CS, Wang XS, Shi Q, Mendoza TR, Wright SL, Berry MD, Malveaux D, Shah PK, Gning I, Hofstetter WL, et al: Automated symptom alerts reduce postoperative symptom severity after cancer surgery: $\mathrm{A}$ randomized controlled clinical trial. J Clin Oncol 2011, 29(8):994-1000.

21. Jenner AV, Mukherjee S, Roberts R, Howe D, Mort D: Patient-reported outcome measures for chemotherapy-related acute toxicity - a single centre experience. Clin Oncol 2010, 22(4):321-322.

22. Yost KJ, Eton DT, Garcia SF, Cella D: Minimally important differences were estimated for six Patient-Reported Outcomes Measurement Information System-Cancer scales in advanced-stage cancer patients. J Clin Epidemiol 2011, 64(5):507-516.
23. Halyard MY: The use of real-time patient-reported outcomes and qualityof-life data in oncology clinical practice. Expert Rev Pharmacoecon Outcomes Res 2011, 11(5):561-570.

24. Fries J, Rose M, Krishnan E: The PROMIS of better outcome assessment: Responsiveness, floor and ceiling effects, and internet administration. J Rheumatol 2011, 38(8):1759-1764

25. Greenhalgh J, Long AF, Flynn R: The use of patient reported outcome measures in routine clinical practice: Lack of impact or lack of theory? Soc Sci Med 2005, 60(4):833-843.

26. Luckett T, Butow PN, King MT: Improving patient outcomes through the routine use of patient-reported data in cancer clinics: Future directions. Psychooncology 2009, 18(11):1129-1138.

27. Marshall S, Haywood K, Fitzpatrick R: Impact of patient-reported outcome measures on routine practice: A structured review. J Eval Clin Pract 2006, 12(5):559-568.

28. Valderas JM, Kotzeva A, Espallargues M, Guyatt G, Ferrans CE, Halyard MY, Revicki DA, Symonds T, Parada A, Alonso J: The impact of measuring patient-reported outcomes in clinical practice: A systematic review of the literature. Qual Life Res 2008, 17(2):179-193.

29. Espallargues M, Valderas JM, Alonso J: Provision of feedback on perceived health status to health care professionals: A systematic review of its impact. Medical Care 2000, 38(2):175-186.

30. Greenhalgh J, Meadows $K$ : The effectiveness of the use of patient-based measures of health in routine practice in improving the process and outcomes of patient care: A literature review. J Eval Clin Pract 1999, 5(4):401-416.

31. Gillbody SM, House AO, Sheldon TA: Outcome measures and needs assessment tools for schizophrenia and related disorders. Cochrane Database Syst Rev 2003, 1, CD003081.

32. Drury M, Yudkin P, Harcourt J, Fitzpatrick R, Jones L, Alcock C, Minton M: Patients with cancer holding their own records: A randomised controlled trial. Br J Gen Pract 2000, 50(451):105-110.

33. Taenzer P, Bultz BD, Carlson LE, Speca M, DeGagne T, Olson K, Doll R, Rosberger Z: Impact of computerized quality of life screening on physician behaviour and patient satisfaction in lung cancer outpatients. Psychooncology 2000, 9(3):203-213.

34. Rosenbloom SK, Victorson DE, Hahn EA, Peterman AH, Cella D: Assessment is not enough: A randomized controlled trial of the effects of HRQL assessment on quality of life and satisfaction in oncology clinical practice. Psychooncology 2007, 16(12):1069-1079.

35. Boyes A, Newell S, Girgis A, McElduff P, Sanson-Fisher R: Does routine assessment and real-time feedback improve cancer patients' psychosocial well-being? Eur J Cancer Care 2006, 15(2):163-171

36. Velikova G, Booth L, Smith AB, Brown PM, Lynch P, Brown JM, Selby PJ: Measuring quality of life in routine oncology practice improves communication and patient well-being: A randomized controlled trial. J Clin Oncol 2004, 22(4):714-724.

37. Detmar SB, Muller MJ, Schornagel JH, Wever LDV, Aaronson NK: Healthrelated quality-of-life assessments and patient-physician communication: A randomized controlled trial. J Am Med Assoc 2002, 288(23):3027-3034.

38. McLachlan SA, Allenby A, Matthews J, Wirth A, Kissane D, Bishop M, Beresford J, Zalcberg J: Randomized trial of coordinated psychosocial interventions based on patient self-assessments versus standard care to improve the psychosocial functioning of patients with cancer. J Clin Oncol 2001, 19(21):4117-4125.

39. Trowbridge R, Dugan W, Jay SJ, Littrell D, Casebeer LL, Edgerton $S$, Anderson J, O'Toole JB: Determining the effectiveness of a clinicalpractice intervention in improving the control of pain in outpatients with cancer. Acad Med 1997, 72(9):798-800.

40. Abernethy AP, Ahmad A, Zafar SY, Wheeler JL, Reese JB, Lyerly HK: Electronic patient-reported data capture as a foundation of rapid learning cancer care. Med Care 2010, 48(6 Suppl):S32-S38.

41. Basch E, Artz D, Dulko D, Scher K, Sabbatini P, Hensley M, Mitra N, Speakman J, McCabe M, Schrag D: Patient online self-reporting of toxicity symptoms during chemotherapy. J Clin Oncol 2005, 23(15):3552-3561.

42. Hoekstra J, De Vos R, Van Duijn NP, Schadé E, Bindels PJE: Using the symptom monitor in a randomized controlled trial: The effect on symptom prevalence and severity. J Pain Symptom Manage 2006, 31(1):22-30

43. Kornblith AB, Dowell JM, Herndon li JE, Engelman BJ, Bauer-Wu S, Small EJ, Morrison VA, Atkins J, Cohen HJ, Holland JC: Telephone monitoring of distress in patients aged 65 years or older with advanced stage cancer: A Cancer and Leukemia Group B study. Cancer 2006, 107(11):2706-2714. 
44. Basch E, lasonos A, Barz A, Culkin A, Kris MG, Artz D, Fearn P, Speakman J, Farquhar R, Scher HI, et al: Long-term toxicity monitoring via electronic patient-reported outcomes in patients receiving chemotherapy. J Clin Oncol 2007, 25(34):5374-5380.

45. Weaver A, Young AM, Rowntree J, Townsend N, Pearson S, Smith J, Gibson O, Cobern W, Larsen M, Tarassenko L: Application of mobile phone technology for managing chemotherapy-associated side-effects. Ann Oncol 2007, 18(11):1887-1892.

46. Butt Z, Wagner LI, Beaumont JL, Paice JA, Straus JL, Peterman AH, Carro G, Von Roenn JH, Shevrin D, Cella D: Longitudinal screening and management of fatigue, pain, and emotional distress associated with cancer therapy. Support Care Cancer 2008, 16(2):151-159.

47. Given CW, Sikorskii A, Tamkus D, Given B, You M, McCorkle R, Champion V, Decker D: Managing symptoms among patients with breast cancer during chemotherapy: Results of a two-arm behavioral trial. J Clin Oncol 2008, 26(36):5855-5862.

48. Hilarius DL, Kloeg PH, Gundy CM, Aaronson NK: Use of health-related qualityof-life assessments in daily clinical oncology nursing practice: A community hospital-based intervention study. Cancer 2008, 113(3):628-637.

49. Mark TL, Fortner B, Johnson G: Evaluation of a tablet PC technology to screen and educate oncology patients. Support Care Cancer 2008, 16(4):371-378.

50. Kearney N, McCann L, Norrie J, Taylor L, Gray P, McGee-Lennon M, Sage M, Miller M, Maguire R: Evaluation of a mobile phone-based, advanced symptom management system (ASyMSC) in the management of chemotherapy-related toxicity. Support Care Cancer 2009, 17(4):437-444.

51. Carlson LE, Groff SL, Maciejewski O, Bultz BD: Screening for distress in lung and breast cancer outpatients: A randomized controlled trial. J Clin Oncol 2010, 28(33):4884-4891.

52. Dinkel A, Berg P, Pirker C, Geinitz H, Sehlen S, Emrich M, Marten-Mittag B, Henrich G, Book K, Herschbach P: Routine psychosocial distress screening in radiotherapy: Implementation and evaluation of a computerised procedure. Br J Cancer 2010, 103(10):1489-1495.

53. Ruland CM, Holte HH, Røislien J, Heaven C, Hamilton GA, Kristiansen J, Sandbæk H, Kvaløy SO, Hasund L, Ellison MC: Effects of a computersupported interactive tailored patient assessment tool on patient care, symptom distress, and patients' need for symptom management support: A randomized clinical trial. J Am Med Inform Assoc 2010, 17(4):403-410.

54. Velikova G, Keding A, Harley C, Cocks K, Booth L, Smith AB, Wright P, Selby PJ, Brown JM: Patients report improvements in continuity of care when quality of life assessments are used routinely in oncology practice: Secondary outcomes of a randomised controlled trial. Eur J Cancer 2010, 46(13):2381-2388.

55. Bainbridge BD, Seow H, Sussman J, Pond G, Martelli-Reid L, Herbert C, Evans W: Multidisciplinary health care professionals' perceptions of the use and utility of a symptom assessment system for oncology patients. Journal of Oncology Practice 2011, 7(1):19-23.

56. Berry DL, Blumenstein BA, Halpenny B, Wolpin S, Fann JR, Austin-Seymour M, Bush N, Karras BT, Lober WB, McCorkle R: Enhancing patient-provider communication with the electronic self-report assessment for cancer: A randomized trial. J Clin Oncol 2011, 29(8):1029-1035.

57. Takeuchi EE, Keding A, Awad N, Hofmann U, Campbell LJ, Selby PJ, Brown JM, Velikova G: Impact of patient-reported outcomes in oncology: A longitudinal analysis of patient-physician communication. $J$ Clin Oncol 2011, 29(21):2910-2917.

58. Grimshaw J, McAuley LM, Bero LA, Grilli R, Oxman AD, Ramsay C, Vale L, Zwarenstein M: Systematic reviews of the effectiveness of quality improvement strategies and programmes. Qual Saf Health Care 2003, 12(4):298-303.

59. Methodological standards for the conduct of Cochrane Intervention Reviews: Cochrane Effective Practice and Organisation of Care Review Group. 2002. http://epoc.cochrane.org/epoc-resources; Accessd 2012.

60. Guyatt GHO, Andrew D, Gunn E, Kunz R, Falck-Ytter Y, Alonso-Coello P. GRADE: an emerging consensus on rating quality of evidence and strength of recommendations. Br Med J 2008, 336:924.

61. Santana MJ, Feeny D, Johnson JA, McAlister FA, Kim D, Weinkauf J, Lien DC: Assessing the use of health-related quality of life measures in the routine clinical care of lung-transplant patients. Qual Life Res 2010, 19(3):371-379.

62. Fayers PM: Evaluating the effectiveness of using PROs in clinical practice: A role for cluster-randomised trials. Qual Life Res 2008, 17(10):1315-1321.
63. Basch E, Abernethy AP: Supporting clinical practice decisions with realtime patient-reported outcomes. J Clin Oncol 2011, 29(8):954-956.

64. Abernethy AP, Wheeler JL, Zafar SY: Management of gastrointestinal symptoms in advanced cancer patients: The rapid learning cancer clinic model. Curr Opin Support Palliat Care 2010, 4(1):36-45.

65. Berry DL: Patient-reported symptoms and quality of life integrated into clinical cancer care. Semin Oncol Nurs 2011, 27(3):203-210.

66. Pawson R, Manzano-Santaella A: A realist diagnostic workshop. Evaluation 2012, 18(2):176-191.

doi:10.1186/1472-6963-13-211

Cite this article as: Chen et al:: A systematic review of the impact of routine collection of patient reported outcome measures on patients, providers and health organisations in an oncologic setting. BMC Health Services Research 2013 13:211.

\section{Submit your next manuscript to BioMed Central and take full advantage of:}

- Convenient online submission

- Thorough peer review

- No space constraints or color figure charges

- Immediate publication on acceptance

- Inclusion in PubMed, CAS, Scopus and Google Scholar

- Research which is freely available for redistribution

Submit your manuscript at www.biomedcentral.com/submit
C Biomed Central 